\title{
Assessment of the Performance of Density Functionals for Predicting Potential Energy Curves in Hydrogen Storage Applications
}

\author{
Srimukh Prasad Veccham and Martin Head-Gordon* \\ Department of Chemistry, University of California, Berkeley, California 94720, USA \\ Chemical Sciences Division, Lawrence Berkeley National Laboratory, Berkeley, California \\ 94720, USA \\ E-mail: mhg@cchem.berkeley.edu
}

\begin{abstract}
The availability of accurate computational tools for modeling and simulation is vital to accelerate the discovery of materials capable of storing hydrogen $\left(\mathrm{H}_{2}\right)$ under given parameters of pressure swing and temperature. Previously, we compiled the H2Bind275 dataset consisting of equilibrium geometries and assessed the performance of 55 density functionals over this dataset (Veccham, S. P.; Head-Gordon, M. J. Chem. Theory Comput., 2020, 16, 4963-4982). As it is crucial for computational tools to accurately model the entire potential energy curve (PEC), in addition to the equilibrium geometry, we have extended this dataset with 389 new data points to include two compressed and three elongated geometries along 78 PECs for $\mathrm{H}_{2}$ binding, forming the $\mathrm{H} 2 \mathrm{Bind} 78 \times 7$ dataset. Assessing the performance of 55 density functionals on this significantly larger and more comprehensive H2Bind $78 \times 7$ dataset, we have identified the best performing density functionals for $\mathrm{H}_{2}$ binding applications: PBE0-DH, $\omega \mathrm{B} 97 \mathrm{X}-\mathrm{V}, \omega \mathrm{B} 97 \mathrm{M}-\mathrm{V}$, and DSD-PBEPBE-D3(BJ). Addition of Hartree Fock exchange improves the performance
\end{abstract}


of density functionals, albeit not uniformly throughout the PEC. We recommend the usage of $\omega \mathrm{B} 97 \mathrm{X}-\mathrm{V}$ and $\omega \mathrm{B} 97 \mathrm{M}-\mathrm{V}$ density functionals as they give good performance for both geometries and energies In addition, we have also identified B97M-V and B97M-rV as the best semi-local density functionals for predicting $\mathrm{H}_{2}$ binding energy at its equilibrium geometry.

\section{Introduction}

Hydrogen $\left(\mathrm{H}_{2}\right)$ is a favorable substitute for fossil fuels as the only by-product of hydrogen fuel cell engines is water and the efficiency of a fuel cell is significantly higher than an internal combustion engine. However, $\mathrm{H}_{2}$ is a light gas with low volumetric and gravimetric energy densities. This poses a significant hurdle to storage and transportation of $\mathrm{H}_{2}$. Storing $\mathrm{H}_{2}$

reversibly in adsorbed form on porous materials is a promising solution to this problem. ${ }^{1-3}$ Ideally, such materials should adsorb $\mathrm{H}_{2}$ at high pressure and release it at low pressure so that the released $\mathrm{H}_{2}$ can be used for operating a fuel cell. Designing materials with this property, while simultaneously not compromising on high volumetric and gravimetric storage capacities, is an active area of research. ${ }^{4,5}$

While multiple porous materials like Metal-Organic Frameworks (MOFs), Covalent Organic Frameworks (COFs), graphene, and other amorphous materials have been shown to adsorb $\mathrm{H}_{2}$, none of these materials meet all the target criteria proposed by the U.S. Department of Energy for an ideal storage material. ${ }^{5}$ As experimental synthesis and characterization of potential $\mathrm{H}_{2}$ storage materials is expensive and time-consuming, computational modeling and screening of materials has emerged as a viable alternative to it. ${ }^{6-8}$ Computational techniques can be used in two different, potentially complementary ways. First, molecular modeling can be used to understand the mechanism of $\mathrm{H}_{2}$ binding in different porous materials and this understanding can be used to systematically tune materials to achieve target properties. ${ }^{5,9,10}$ Second, computational techniques can be used to screen materials in a highthroughput manner to select only a handful of potentially viable materials for synthesis and 
characterization.

The ability of a material to store $\mathrm{H}_{2}$ is characterized by its usable capacity, which is defined as the amount of $\mathrm{H}_{2}$ stored at the high operating pressure that is released when the pressure is reduced to the low operating pressure. Optimizing the usable capacity for typical fixed operating pressures of 5 bar and 100 bar gives an optimal value for the Gibbs free energy of adsorption $\left(\Delta G_{\mathrm{ads}}\right)$. Assuming a correlation between enthalpy and entropy of adsorption in porous materials gives a range of -15 to $-25 \mathrm{~kJ} / \mathrm{mol}$ for the optimal value for enthalpy of adsorption $\left(\Delta H_{\text {ads }}\right) .{ }^{11-13}$ The internal energy of binding, which is the largest component of $\Delta H_{\text {ads }}$, can be computed using different quantum chemistry methods, including, but not limited to, density functional theory (DFT), ${ }^{9,10,14,15}$ Møller-Plesset perturbation theory (MP2), ${ }^{16-18}$ and different variants of coupled-cluster theory. ${ }^{19-21}$ Each of these methods have different accuracies and computational costs associated with them.

DFT, scaling as $\mathcal{O}\left(N^{3}\right)$ ( $N$ is the number of basis functions in the system), can provide a reasonable balance between cost and accuracy of computing $\mathrm{H}_{2}$ binding energy. However, as the exact density functional remains unknown, different density functional approximations (DFAs), proposed in lieu of the exact density functional, provide varying accuracies for different chemical systems and/or properties computed. ${ }^{22}$ In order to address this problem, we adopted a two-pronged approach. ${ }^{21}$ (1) We compiled the H2Bind275 dataset that consists of $\mathrm{H}_{2}(\mathrm{~s})$ interacting with binding motifs representative of different porous materials known for $\mathrm{H}_{2}$ adsorption. This dataset consists only of equilibrium geometries, that is, $\mathrm{H}_{2}(\mathrm{~s})$ are located at the minimum of the potential energy curve (PEC) with respect to the binding site. We computed highly accurate reference interaction energies using coupled-cluster singles, doubles, and perturbative triples $(\operatorname{CCSD}(\mathrm{T}))$ extrapolated to the complete basis set limit for this dataset. (2) We assessed the performance of 55 DFAs and identified the best performing density functionals for this dataset. In addition, we also identified inexpensive semi-local density functionals which give very good performance for low computational cost and are suitable for in silico high-throughput screening purposes. 
The H2Bind275 dataset, consisting of 275 data points, provides a balanced representation of different $\mathrm{H}_{2}$ binding mechanisms like polarization, charge transfer interaction, and dispersion. ${ }^{15,23,24}$ It also captures the chemical diversity of binding motifs that $\mathrm{H}_{2}$ interacts with in porous frameworks. This dataset assesses the ability of density functionals to reproduce $\mathrm{H}_{2}$ binding energies at the minima of the PEC. However, as DFAs are routinely used for geometry optimizations and molecular dynamics simulations either directly or indirectly (by generating reference data for training force fields), they should also be able to reproduce the entire PEC which would ensure accurate nuclear gradients as required for geometry optimization and molecular dynamics simulations. A strategy of assessing the performance of DFAs for PECs has been previously employed for other non-covalent interaction energy datasets like S22, S66, and A24. The S22x5 dataset ${ }^{25}$ was created from the S22 dataset ${ }^{26}$ by including geometries that are shortened and elongated along a well-defined interaction coordinate. Similarly, the S66 $\mathrm{x} 8^{27}$ and $\mathrm{A} 21 \times 12^{28}$ extended datasets were created from the S66 and A24 datasets. ${ }^{29}$

In order to address this issue for $\mathrm{H}_{2}$ storage, we have extended the $\mathrm{H} 2 \mathrm{Bind} 275$ dataset to include geometries that are located at five different points on 78 separate PECs, not just the minimum. This extended dataset, hereafter referred to as the H2Bind $78 \times 7$ dataset, was generated by shortening and stretching the distance between $\mathrm{H}_{2}$ and the binding motif. The reference interaction energies were computed using $\operatorname{CCSD}(\mathrm{T})$ extrapolated to the complete basis set (CBS) limit using the same strategy outlined in Ref. 21. The performance of 55 DFAs were assessed using regularized relative errors metrics by appropriately weighing the error coming from different points on the PECs. We have analyzed the performance of these DFAs for the extended dataset by comparing and contrasting it with the performance of the original equilibrium H2Bind275 dataset.

This paper is organized as follows. The H2Bind $78 \times 7$ dataset is introduced and the protocol for computing reference interaction energies is discussed. All the density functionals chosen for assessment in this work are briefly introduced and classified. The distribution 
of the reference interaction energies at different points on the PEC is outlined. The performance of DFAs for predicting $\mathrm{H}_{2}$ interaction energies across the PEC is discussed and contrasted with their performance for the previous H2Bind275 dataset. The performance of DFAs for predicting equilibrium geometries and interaction energies at equilibrium geometries is explored. The best DFAs for predicting $\mathrm{H}_{2}$ binding energies are recommended while considering their computational cost.

\section{Computational details}

\section{H2Bind $78 \times 7$ dataset}

Table 1: Number of geometries and data points by chemical categories for the H2Bind $78 \times 7$ dataset

\begin{tabular}{cccccc}
\hline & s-block ions & salts & organic ligands & transition metals & total \\
\hline geometries & 19 & 13 & 5 & 41 & 78 \\
data points at PEC minimum & 38 & 26 & 10 & 82 & 156 \\
data points not at PEC minimum & 95 & 65 & 25 & $204^{1}$ & 389 \\
H2Bind78 $\times 7$ & 133 & 91 & 35 & 286 & 545 \\
\hline
\end{tabular}

The H2Bind275 dataset consists of $275 \mathrm{H}_{2}$ interaction energies but only 86 unique geometries as many of them have multiple $\mathrm{H}_{2}$ s. For example, the geometry of $\mathrm{CaCl}_{2}-\left(\mathrm{H}_{2}\right)_{4}$ has four hydrogen molecules bound to $\mathrm{CaCl}_{2}$ contributing four data points to the $\mathrm{H} 2 \mathrm{Bind} 275$ dataset. The H2Bind $78 \times 7$ dataset was generated by starting from a subset of the original H2Bind275 dataset. This subset was created by choosing only 78 unique geometries and computing their interaction energies adiabatically using the method outlined in Ref. 21. All of these geometries are located on the PEC at their respective minima. The adiabatic interaction energy, which relaxes the geometries of the binding motif and $\mathrm{H}_{2}$, was chosen as it is closest to experimentally measurable values. For each minimum geometry, five additional geometries were generated by compressing and elongating the distance between the binding motif and the center of mass of $\mathrm{H}_{2}$ (denoted by $r_{\text {eq }}$ ). For geometries containing multiple

\footnotetext{
${ }^{1}$ One data point excluded due to convergence issues
} 
$\mathrm{H}_{2} \mathrm{~s}$ bound to a single binding moiety, compressed and elongated geometries were generated for only one of the $\mathrm{H}_{2} \mathrm{~s}$. This step was necessary in order to maintain low redundancy in the dataset and make its size manageable. In addition to this, the interaction energy at the minimum of the PEC was also computed using vertical interaction energy method. In total, this dataset contains 78 adiabatic and 78 vertical interaction energies (a total of 156 data points) located at the PEC minimum.

In this work, two compressed geometries $\left(0.75 r_{\text {eq }}\right.$ and $\left.0.9 r_{\text {eq }}\right)$ and three elongated geometries $\left(1.1 r_{\mathrm{eq}}, 1.25 r_{\mathrm{eq}}\right.$, and $\left.1.5 r_{\mathrm{eq}}\right)$ were considered. These distances were chosen as they are representative of the PEC in both the compressed and elongated regimes. In a porous material, $\mathrm{H}_{2}$ interacts with not only its primary binding site but also has secondary interactions with other components of the framework. The binding distances of $\mathrm{H}_{2}$ to its secondary interaction sites of the porous material are often longer than their corresponding equilibrium distances. As a consequence of this, when modeling $\mathrm{H}_{2}$ in a porous material, the elongated portion of the PEC is sampled more than the compressed part. Additionally, the compressed portion of the PEC is usually significantly higher in energy (repulsive if compressed enough), and is sampled less often in a molecular dynamics or Monte Carlo simulation. Hence, DFAs should be able to reproduce the elongated portion of the PEC more faithfully than the compressed portion. We have included more data points in the elongated regime than the compressed regime in order to underscore its relative importance. As shown in Table 1, the number of non-equilibrium data points is roughly 2.5 times the number of data points at equilibrium. In total, counting both the equilibrium and non-equilibrium data points, this $\mathrm{H} 2 \mathrm{Bind} 78 \times 7$ dataset consists of $545 \mathrm{H}_{2}$ interaction energies with representative binding motifs.

This dataset, like the H2Bind275 dataset, can also be divided into categories based on the chemical nature of the binding motif as shown in Table 2: (1) s-block ions: consisting of group 1 and group 2 bare metal cations with unscreened charge binding one or multiple $\mathrm{H}_{2} \mathrm{~s},(2)$ salts: consisting of small inorganic salts like $\mathrm{AlF}_{3}, \mathrm{CaCl}_{2}$, and $\mathrm{MgF}_{2}$ binding one or multiple 
Table 2: All 78 geometries in the H2Bind $78 \times 7$ dataset categorized by chemical identity of the binding motif.

\begin{tabular}{c|c|c|c}
\hline s-block ions & salts & organic ligands & transition metals \\
\hline $\mathrm{Li}^{+}-\left(\mathrm{H}_{2}\right)_{\mathrm{n}}$, & $\mathrm{AlF}_{3}-\mathrm{H}_{2}$ & benzene- $\mathrm{H}_{2}$ & $\mathrm{MX}-\mathrm{H}_{2}, \mathrm{X}=\mathrm{H}, \mathrm{F}, \mathrm{Cl} ; \mathrm{M}=\mathrm{Cu}, \mathrm{Ag}, \mathrm{Au}$ \\
$n=1,2,3,4,5,6$ & $\mathrm{CaF}_{2}-\left(\mathrm{H}_{2}\right)_{\mathrm{n}}$, & phenol- $\mathrm{H}_{2}$ & $\mathrm{CoF}_{3}-\mathrm{H}_{2}$ \\
$\mathrm{Na}^{+}-\left(\mathrm{H}_{2}\right)_{\mathrm{n}}$, & $n=1,2,3,4$ & pyrrole- $\mathrm{H}_{2}$ & $\mathrm{Cu}(\mathrm{OMe})-\mathrm{H}_{2}$ \\
$n=1,2,3$ & $\mathrm{CaCl}_{2}-\left(\mathrm{H}_{2}\right)_{\mathrm{n}}$, & butene- $\mathrm{H}_{2}$ & $\mathrm{CuCN}-\mathrm{H}_{2}$ \\
$\mathrm{Mg}^{2+}-\left(\mathrm{H}_{2}\right)_{\mathrm{n}}$, & $n=1,2,3,4$ & tetrazole- $\mathrm{H}_{2}$ & $\mathrm{Sc}^{+}-\left(\mathrm{H}_{2}\right)_{\mathrm{n}}, \mathrm{V}^{+}-\left(\mathrm{H}_{2}\right)_{\mathrm{n}}, n=3,4$ \\
$n=1,2,3,4$ & $\mathrm{MgF}_{2}-\left(\mathrm{H}_{2}\right)_{\mathrm{n}}$, & & $\mathrm{Ti}^{+}-\left(\mathrm{H}_{2}\right)_{\mathrm{n}}, n=2,4$ \\
$\mathrm{Ca}^{2+}-\left(\mathrm{H}_{2}\right)_{\mathrm{n}}$, & $n=1,2,3,4$ & & $\mathrm{Cr}^{+}-\left(\mathrm{H}_{2}\right)_{\mathrm{n}}, \mathrm{Mn}^{+}-\left(\mathrm{H}_{2}\right)_{\mathrm{n}}, n=1,2,3,4$ \\
$n=1,2,3,4,5,6$ & & $\mathrm{Fe}^{+}-\left(\mathrm{H}_{2}\right)_{\mathrm{n}}, n=1,2,3,4$ \\
& & $\mathrm{Co}^{+}-\left(\mathrm{H}_{2}\right)_{\mathrm{n}}, \mathrm{Ni}^{+}-\left(\mathrm{H}_{2}\right)_{\mathrm{n}}, n=1,2$ \\
& & $\mathrm{Cu}^{+}-\left(\mathrm{H}_{2}\right)_{\mathrm{n}}, n=1,2,3$ \\
& & $\mathrm{Zn}^{+}-\left(\mathrm{H}_{2}\right)_{\mathrm{n}}, n=1,2,3,4$ \\
\hline
\end{tabular}

$\mathrm{H}_{2} \mathrm{~s}$, (3) organic ligands: comprising of small aliphatic and aromatic molecules binding one $\mathrm{H}_{2}$, (4) transition metals: including small transition metal complexes and $3 \mathrm{~d}$ transition metal cations binding one or multiple $\mathrm{H}_{2} \mathrm{~s}$. Each of these categories is also representative of various mechanisms of $\mathrm{H}_{2}$ binding found in porous materials. For example, $\mathrm{H}_{2}$ in the organic ligands category is mostly dispersion-bound. The s-block metals category binds $\mathrm{H}_{2}$ using a combination of electrostatic and forward charge transfer $\left(\mathrm{H}_{2} \rightarrow\right.$ metal $)$ interactions. ${ }^{15}$ This dataset captures both chemical and mechanistic diversity encountered in $\mathrm{H}_{2}$ binding to porous materials. For a detailed discussion about different chemical categories in this dataset, we refer readers to Ref. 21.

\section{Reference Binding Energies}

Calculation of accurate reference interaction energies is an important task in compiling a dataset. Reference interaction energies were computed using coupled-cluster theory with singles, doubles, and perturbative triples $(\operatorname{CCSD}(\mathrm{T}))^{30}$ extrapolated to the complete basis set limit. Inspired by the success of composite extrapolation methods ${ }^{31-34}$ for computing highly accurate reference values, we have developed our own composite extrapolation method 
using focal point analysis ${ }^{35,36}$ for computing accurate reference $\mathrm{H}_{2}$ binding energies:

$$
\begin{aligned}
E_{\mathrm{ref}} & =E_{\mathrm{HF} / 5 \mathrm{Z}}+E_{\mathrm{MP} 2 / \mathrm{QZ} \rightarrow 5 \mathrm{Z}}+\delta E_{\mathrm{CCSD}(\mathrm{T}) / \mathrm{TZ}}+\delta E_{\mathrm{MP} 2 / \mathrm{TZ}}^{\mathrm{core}} \\
\delta E_{\mathrm{CCSD}(\mathrm{T}) / \mathrm{TZ}} & =E_{\mathrm{CCSD}(\mathrm{T}) / \mathrm{TZ}}-E_{\mathrm{MP} 2 / \mathrm{TZ}} \\
\delta E_{\mathrm{MP} 2 / \mathrm{TZ}}^{\mathrm{core}} & =E_{\mathrm{MP} 2 / \mathrm{TZ}}^{\mathrm{core}=0}-E_{\mathrm{MP} 2 / \mathrm{TZ}}^{\mathrm{core}=\mathrm{n}}
\end{aligned}
$$

Here, $E_{\text {ref }}$ is the reference energy computed using the composite method, $E_{\mathrm{HF} / 5 \mathrm{Z}}$ is the Hartree Fock energy computed using a basis set of quintuple-zeta (5Z) quality, $E_{\mathrm{MP} 2 / \mathrm{QZ} \rightarrow 5 \mathrm{Z}}$ is the MP2 correlation energy extrapolated to the complete basis set limit with the 2-point extrapolation formula ${ }^{37}$ using correlation energies computed with quadruple-zeta (QZ) and quintuple-zeta quality basis sets, and $\delta E_{\operatorname{CCSD}(\mathrm{T}) / \mathrm{TZ}}$ is the difference between the $\operatorname{CCSD}(\mathrm{T})$ and MP2 correlation energies computed with a triple-zeta quality basis set. $\delta E_{\mathrm{MP} 2 / \mathrm{Tz}}^{\mathrm{core}}$ is the core-valence contribution to the correlation energy computed as the difference between MP2 correlation energies with $\left(E_{\mathrm{MP} 2 / \mathrm{TZ}}^{\mathrm{core}=\mathrm{n}}\right)$ and without $\left(E_{\mathrm{MP} 2 / \mathrm{TZ}}^{\mathrm{core}=0}\right)$ the frozen-core approximation. This composite method for computing reference $\mathrm{H}_{2}$ interaction energies ensures that the effect of higher-order excitations neglected in $\operatorname{CCSD}(\mathrm{T})$ are sufficiently small. It also ensures that the basis set incompleteness errors are small and that both HF and extrapolated correlation energy components are of complete basis set limit quality. Further details of this scheme can be found in Ref. 21.

The cc-pVnZ ${ }^{38,39}(n=\mathrm{T}, \mathrm{Q}$, or 5) family of basis sets was used for all the HF and correlation energy calculations when core electrons were not included in the correlation calculations. cc-pCVnZ ${ }^{40,41}(n=\mathrm{T}, \mathrm{Q}$, or 5) family of basis sets were employed when some or all of the core electrons were included in the correlation calculations. For transition metals, the cc-pwCVnZ ${ }^{42}(n=\mathrm{T}, \mathrm{Q}$, or 5$)$ series of basis sets was used with a neon core excluded in all correlation energy computations. 


\section{Density Functional Approximations}

55 DFAs, including all the commonly used density functionals, were chosen to perform a thorough assessment. We have also included DFAs that have previously shown very good performance for a range of non-covalent interaction energy prediction problems represented by multiple datasets. ${ }^{43}$ Based on the different quantities DFAs depend on, they are categorized into rungs of the metaphorical Jacob's ladder. ${ }^{44}$ From the first rung of the Jacob's ladder, in which DFAs depend only on electron density, SVWN5 ${ }^{45,46}$ and SPW92 ${ }^{45,47}$ DFAs were chosen. From the second rung called Generalized Gradient Approximation (GGA), 12 different DFAs were chosen: the PBE family and its variants (PBE, ${ }^{48}$ PBE-D3(0), ${ }^{49}$ RPBE,${ }^{50}$ revPBE ${ }^{51}$ and revPBE-D3(op) ${ }^{52}$ ), BLYP ${ }^{53,54}$ and BLYP-D3(op) ${ }^{52}$, dispersioncorrected variants of B97 ${ }^{55}$ (BLYP-D3(0) ${ }^{49}$ and BLYP-D3(BJ) ${ }^{56}$ ), PW91, ${ }^{57}$ and GAM. ${ }^{58}$ From the meta-GGA rung, the different variants of TPSS (TPSS, ${ }^{59}$ TPSS-D3(BJ) ${ }^{56}$ and revTPSS $\left.{ }^{60}\right), \mathrm{SCAN}^{61}$ and its dispersion-corrected version SCAN-D3(BJ) ${ }^{62}, \mathrm{MS}^{63}$ and MS2D3(op), ${ }^{52}$ the combinatorially-optimized B97M-V ${ }^{64}$ and B97M-rV ${ }^{65,66}$ were chosen. In addition, mBEEF ${ }^{67}$ and the semi-local Minnesota functionals M06- ${ }^{68}$ and MN15-L ${ }^{69}$ were also included in the assessment. Rung four DFAs, containing HF exchange, are generally more accurate than semi-local functionals as they partially alleviate the problem of self-interaction

error. In this work, global hybrid density functionals like B3LYP ${ }^{70}$ and B3LYP-D3(0), ${ }^{56}$ PBE0 ${ }^{71}$ and PBE0-D3(BJ), ${ }^{56}$ TPSSh $^{72}$ and TPSSh-D3(BJ), ${ }^{56}$ the M06 family of density functionals (M06, ${ }^{73}$ M06-2X, ${ }^{73}$ M06-2X-D3(0), ${ }^{49}$ and revM06 ${ }^{74}$ ), MVSh, ${ }^{75}$ and SCAN0 ${ }^{76}$ which is the hybrid variant of SCAN are included. Range-separated hybrids, which are hybrid functionals containing DFT exchange and some HF exchange in the short-range and only HF exchange in the long range, included in this study are $\omega \mathrm{B} 97 \mathrm{X}-\mathrm{D},{ }^{77} \omega \mathrm{B} 97 \mathrm{X}-\mathrm{D} 3,{ }^{78}$ $\omega \mathrm{B} 97 \mathrm{X}-\mathrm{V},{ }^{79} \omega \mathrm{B} 97 \mathrm{M}-\mathrm{V},{ }^{22} \mathrm{M} 11^{80}$ and its revised version revM11. ${ }^{81}$ Two screened exchange density functionals (HSE-HJS ${ }^{82,83}$ and MN12-SX ${ }^{84}$ ), which contain DFT exchange in the short range and attenuated HF exchange in the long range are also included. Double hybrid density functionals, which are at the top the Jacob's ladder classification, contain some 
percentage of correlation energy from wavefunction methods. These DFAs are characterized by their superior accuracy and increased computational cost in comparison to semi-local and hybrid DFAs. We have included seven double hybrid density functionals in this study: B2PLYP-D3(BJ) ${ }^{85}$ XYG3 ${ }^{86}$ XYGJ-OS, ${ }^{87}$ PBE0-DH,${ }^{88}$, PTPSS-D3(0), ${ }^{89}$ DSD-PBEPBED3(BJ), ${ }^{90}$ and $\omega \mathrm{B} 97 \mathrm{M}(2) .{ }^{91}$

The def2-QZVPPD ${ }^{92}$ basis set was used for all DFA calculations with a quadrature grid of 99 Euler-MacLaurin radial points and 590 Lebedev angular points for integrating the exchange-correlation contribution. SG- ${ }^{93}$ integration grid was used for integrating the VV10 component. The choice of core for frozen core approximation and employment of density fitting approximation for computing MP2 correlation energy in double hybrid density functionals is discussed in Table S1. All the PECs were interpolated using the one-dimensional Akima interpolator. ${ }^{94}$ All computations were performed using Q-Chem 5. ${ }^{95}$

\section{Results and Discussion}

\section{H2Bind $78 \times 7$ dataset}

Typically, the coupled cluster reference $\mathrm{H}_{2}$ interaction energy with the binding motif is strongest at equilibrium, that is at $r_{\text {eq }}$. This implies that the geometries optimized using $\omega B 97 M-V /$ def2-TZVPD ${ }^{92}$ are also close to the CCSD(T)/CBS minima. Fig. 1 shows the distribution of interaction energies for the entire H2Bind $78 \times 7$ dataset consisting of 545 data points. The equilibrium and geometries near equilibrium $\left(0.9 r_{\mathrm{eq}}, 1.0 r_{\mathrm{eq}}, 1.0 r_{\mathrm{eq}}^{\mathrm{vert}}\right.$, and $\left.1.1 r_{\mathrm{eq}}\right)$ have attractive interaction energies, with most of them smaller than $100 \mathrm{~kJ} / \mathrm{mol}$ in magnitude. Geometries that are stretched by $25 \%\left(1.25 r_{\text {eq }}\right)$ are still attractive in nature, but most interaction energies are smaller than $60 \mathrm{~kJ} / \mathrm{mol}$ in magnitude. Geometries stretched by $50 \%$ of their equilibrium distance are bound only weakly with a median binding energy of $-6.9 \mathrm{~kJ} / \mathrm{mol}$. At the other extreme of the PEC, geometries that are compressed by

$25 \%\left(0.75 r_{\text {eq }}\right)$ are mostly repulsive with a median interaction energy of $+29.7 \mathrm{~kJ} / \mathrm{mol}$. This 


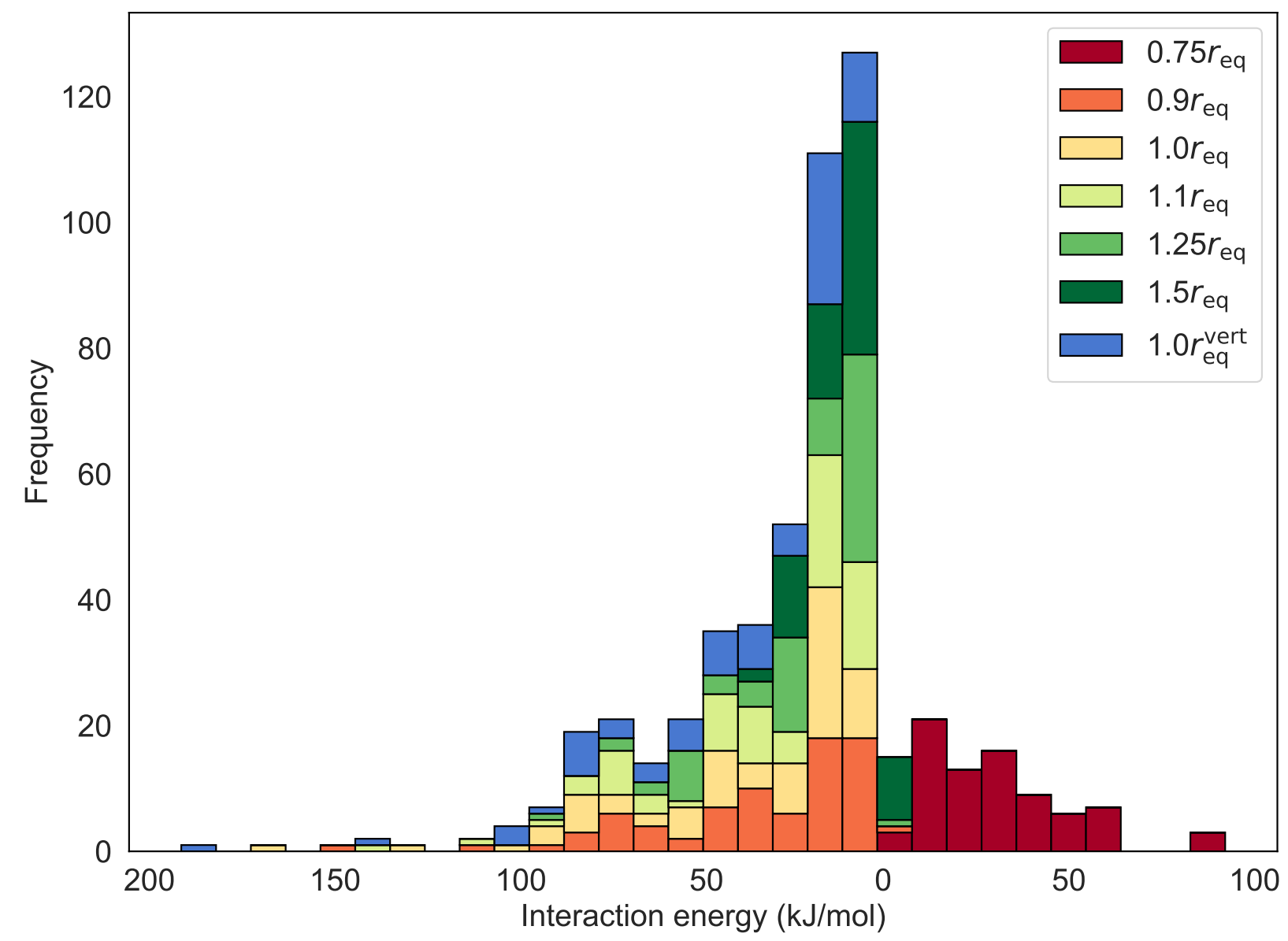

Figure 1: Distribution of coupled-cluster reference interaction energies separated by location on the potential energy curve. The reference vertical interaction energy at equilibrium $\left(1.0 r_{\mathrm{eq}}^{\mathrm{vert}}\right)$ is also shown. 
geometry was also chosen in order to sample the repulsive part of the PEC and assess how accurately different density functionals can reproduce it. ${ }^{96}$

The range of interaction energies covered by each PEC is also very large. The coinage metal containing species are the strongest binders, as illustrated by the extreme example of $\mathrm{AuF}$ which binds $\mathrm{H}_{2}$ with an interaction energy of $-161.8 \mathrm{~kJ} / \mathrm{mol}$ at equilibrium and interacts with $\mathrm{H}_{2}$ with an energy of $+87.6 \mathrm{~kJ} / \mathrm{mol}$ (repulsive) at $0.75 r_{\text {eq }}$, thus spanning an interaction range of $249.4 \mathrm{~kJ} / \mathrm{mol}$. Data points in the organic category have the smallest ranges (average range is $19 \mathrm{~kJ} / \mathrm{mol}$ ). A typical $\mathrm{PEC}$ of $\mathrm{H}_{2}$ interacting with a binding moiety has the shape of a Morse potential. However, there is considerable variation in the well depth, well width, and decay in the long range for different chemical species. This variation can provide some clues into the dominant mechanism of interaction. For example, the $\mathrm{AuCl}$ binding motif interacts with one $\mathrm{H}_{2}$ with an interaction energy of $-123.7 \mathrm{~kJ} / \mathrm{mol}$ at equilibrium which decays to $-21.3 \mathrm{~kJ} / \mathrm{mol}$ at $1.5 r_{\text {eq }}(82.7 \%$ decrease $)$. This is a sharp decay in the interaction

energy in comparison to the $\mathrm{Mg}^{2+}$ case. In the $\mathrm{Mg}^{2+}$ interacting with one $\mathrm{H}_{2}$ case, the interaction energy at equilibrium is $-97.8 \mathrm{~kJ} / \mathrm{mol}$ in comparison to $-37.7 \mathrm{~kJ} / \mathrm{mol}$ at $1.5 r_{\text {eq }}$ (61.4\% decrease). This suggested that the dominant mechanism of interaction in $\mathrm{Mg}^{2+}$ case is longer-ranged (like permanent electrostatics) than $\mathrm{AuCl}$ which is dominated by orbital controlled short-ranged interactions like charge transfer.

\section{Performance of Density Functional Approximations on PECs}

We will discuss the performance of DFAs using multiple error metrics. Each of these metrics gives different weights to different aspects of the dataset. First, we will discuss the performance of DFAs using the root mean square error (RMSE) metric which gives equal importance to all data points in the H2Bind $78 \times 7$ dataset. The RMSE of all 55 DFAs assessed in this work is shown in Table 3. The non-empirical double hybrid functional with just two fixed parameters, PBE0-DH, gives the least RMSE of $2.9 \mathrm{~kJ} / \mathrm{mol}$. The second best DFA is another double hybrid DSD-PBEPBE-D3(BJ) with an RMSE of $3.7 \mathrm{~kJ} / \mathrm{mol}$. In com- 
Table 3: Regularized mean absolute percentage error (RegMAPE) and root mean squared error (RMSE; in $\mathrm{kJ} / \mathrm{mol}$ ) of all DFAs considered in this work for the entire $\mathrm{H} 2 \mathrm{Bind} 78 \times 7$ dataset.

\begin{tabular}{|c|c|c|c|c|}
\hline Rank & DFA & RMSE $(\mathrm{kJ} / \mathrm{mol})$ & DFA & RegMAPE (\%) \\
\hline 1 & PBE0-DH & 2.9 & PBE0-DH & 5.0 \\
\hline 2 & DSD-PBEPBE-D3(BJ) & 3.7 & $\omega \mathrm{B} 97 \mathrm{X}-\mathrm{V}$ & 5.4 \\
\hline 3 & $\omega \mathrm{B} 97 \mathrm{X}-\mathrm{V}$ & 4.0 & $\omega \mathrm{B} 97 \mathrm{M}-\mathrm{V}$ & 6.3 \\
\hline 4 & $\omega \mathrm{B} 97 \mathrm{X}-\mathrm{D}$ & 4.1 & DSD-PBEPBE-D3(BJ) & 6.3 \\
\hline 5 & PBE0 & 4.2 & XYGJ-OS & 6.9 \\
\hline 6 & MVSh & 4.3 & $\omega \mathrm{B} 97 \mathrm{M}(2)$ & 7.4 \\
\hline 7 & HSE-HJS & 4.3 & PBE0 & 7.6 \\
\hline 8 & $\omega \mathrm{B} 97 \mathrm{M}-\mathrm{V}$ & 4.5 & HSE-HJS & 7.6 \\
\hline 9 & XYGJ-OS & 4.6 & B2PLYP-D3(BJ) & 8.2 \\
\hline 10 & $\omega \mathrm{B} 97 \mathrm{X}-\mathrm{D} 3$ & 4.8 & XYG3 & 8.5 \\
\hline 11 & XYG3 & 4.8 & $\omega \mathrm{B} 97 \mathrm{X}-\mathrm{D}$ & 9.0 \\
\hline 12 & PTPSS-D3(0) & 4.9 & B97M-rV & 9.0 \\
\hline 13 & $\omega \mathrm{B} 97 \mathrm{M}(2)$ & 5.1 & B97M-V & 9.1 \\
\hline 14 & PBE0-D3(BJ) & 5.2 & SCANO & 9.1 \\
\hline 15 & MN15 & 5.7 & PTPSS-D3(0) & 9.3 \\
\hline 16 & B2PLYP-D3(BJ) & 5.8 & $\omega \mathrm{B} 97 \mathrm{X}-\mathrm{D} 3$ & 9.8 \\
\hline 17 & SCAN0 & 5.8 & MVSh & 10.2 \\
\hline 18 & TPSSh & 6.0 & TPSSh & 11.3 \\
\hline 19 & revM11 & 6.3 & PBE0-D3(BJ) & 11.5 \\
\hline 20 & mBEEF & 6.7 & M11 & 12.0 \\
\hline 21 & revM06 & 6.9 & revTPSS & 12.0 \\
\hline 22 & B3LYP & 7.4 & revM06 & 12.4 \\
\hline 23 & revTPSS & 7.5 & TPSS & 13.6 \\
\hline 24 & B3LYP-D3(0) & 7.5 & TPSSh-D3(BJ) & 13.8 \\
\hline 25 & B97M-V & 7.6 & B3LYP-D3(0) & 14.0 \\
\hline 26 & B97M-rV & 7.6 & oTPSS-D3(BJ) & 14.6 \\
\hline 27 & TPSS & 7.7 & TPSS-D3(BJ) & 14.7 \\
\hline 28 & oTPSS-D3(BJ) & 7.8 & MN15 & 15.3 \\
\hline 29 & TPSSh-D3(BJ) & 8.0 & $\mathrm{PBE}$ & 15.3 \\
\hline 30 & MN15-L & 8.0 & revM11 & 15.3 \\
\hline 31 & revPBE-D3(op) & 8.5 & BLYP-D3(op) & 15.4 \\
\hline 32 & TPSS-D3(BJ) & 8.5 & B3LYP & 15.5 \\
\hline 33 & revPBE & 9.1 & SCAN & 15.6 \\
\hline 34 & MN12-SX & 9.2 & MN12-SX & 16.2 \\
\hline 35 & RPBE & 9.3 & SCAN-D3(BJ) & 16.3 \\
\hline 36 & M11 & 9.4 & M06 & 16.9 \\
\hline 37 & B97-D3(BJ) & 9.7 & revPBE-D3(op) & 17.0 \\
\hline 38 & BLYP-D3(op) & 9.8 & PW91 & 17.4 \\
\hline 39 & M06 & 9.9 & MS2 & 17.4 \\
\hline 40 & BLYP & 10.1 & mBEEF & 17.4 \\
\hline 41 & PBE & 10.3 & BP86-D3(BJ) & 18.4 \\
\hline 42 & M06-L & 10.5 & M06-2X & 19.3 \\
\hline 43 & BP86-D3(BJ) & 10.9 & M06-2X-D3(0) & 19.8 \\
\hline 44 & B97-D3(0) & 11.0 & MN15-L & 19.8 \\
\hline 45 & PBE-D3(0) & 11.0 & PBE-D3(0) & 20.1 \\
\hline 46 & PW91 & 11.2 & MS2-D3(op) & 20.3 \\
\hline 47 & GAM & 11.8 & M06-L & 20.7 \\
\hline 48 & MS2 & 12.1 & RPBE & 20.9 \\
\hline 49 & MS2-D3(op) & 12.4 & BLYP & 22.1 \\
\hline 50 & SCAN & 12.9 & revPBE & 23.4 \\
\hline 51 & SCAN-D3(BJ) & 13.2 & B97-D3(BJ) & 24.2 \\
\hline 52 & M06-2X & 13.4 & GAM & 24.7 \\
\hline 53 & M06-2X-D3(0) & 13.4 & B97-D3(0) & 28.3 \\
\hline 54 & SPW92 & 32.6 & SPW92 & 63.0 \\
\hline 55 & SVWN5 & 32.7 & SVWN5 & 63.0 \\
\hline
\end{tabular}


parison to the earlier H2Bind275 dataset where DSD-PBEPBE-D3(BJ) was ranked fourth, it performs relatively better for the H2Bind $78 \times 7$ dataset moving up by two places. ${ }^{21}$ This is closely followed by $\omega$ B97X-V and $\omega$ B97X-D, both of which show a similar RMSEs of 4.0 and $4.1 \mathrm{~kJ} / \mathrm{mol}$ respectively. Another trend seen in the H2Bind275 dataset that is transferable to the H2Bind $78 \times 7$ dataset is that the best performing DFA in each rung of the Jacob's ladder performs better than the best performing functional in rung directly below it. The least RMSE DFA in each rung also remains the same: SPW92 for LDA, revPBE-D3(op) for GGAs, mBEEF for meta-GGAs, $\omega$ B97X-V in the hybrids rung, and PBE0-DH in the double hybrids rung. The ranking of $\omega \mathrm{B} 97 \mathrm{M}-\mathrm{V}$ deteriorates in the extended dataset in comparison to the previous H2Bind275 dataset. Another interesting observation is the improvement in the ranking of the MN15 density functional which is ranked $15^{\text {th }}$ in the H2Bind $78 \times 7$ dataset with an RMSE of $5.7 \mathrm{~kJ} / \mathrm{mol}$ (MN15 was ranked $25^{\text {th }}$ with an RMSE of $6.3 \mathrm{~kJ} / \mathrm{mol}$ in the H2Bind275 dataset). The relative performance of B97M-V and B97M-rV (ranked $25^{\text {th }}$ and $\left.26^{\text {th }}\right)$ in the H2Bind $78 \times 7$ dataset remains comparable to their performance in the H2Bind275 dataset. We also note that commonly used density functionals like M06-2X and M06-2XD3(0) and the recently developed density functionals like SCAN and SCAN-D3(BJ) show very large RMSEs.

The reference interaction energies in the $\mathrm{H} 2 \mathrm{Bind} 78 \times 7$ dataset span a very large range: from -189.0 to $92.1 \mathrm{~kJ} / \mathrm{mol}$. However, for $\mathrm{H}_{2}$ storage applications between 5 and 100 bar, interaction energies in the -15 to $-25 \mathrm{~kJ} / \mathrm{mol}$ range would be ideal. ${ }^{11-13}$ A good error metric should give more weight to data points in this range by considering the following aspects:

1. The H2Bind $78 \times 7$ dataset contains many model binding motifs, each of them binding $\mathrm{H}_{2}$ with different interaction energies at their corresponding equilibrium geometry. Binding motifs that bind $\mathrm{H}_{2}$ with an interaction energy in the range of -15 to -25 $\mathrm{kJ} / \mathrm{mol}$ should have larger weights.

2. Each binding motif contributes six data points: two compressed data points, three elongated data points, and one data point at equilibrium. Equilibrium geometries 


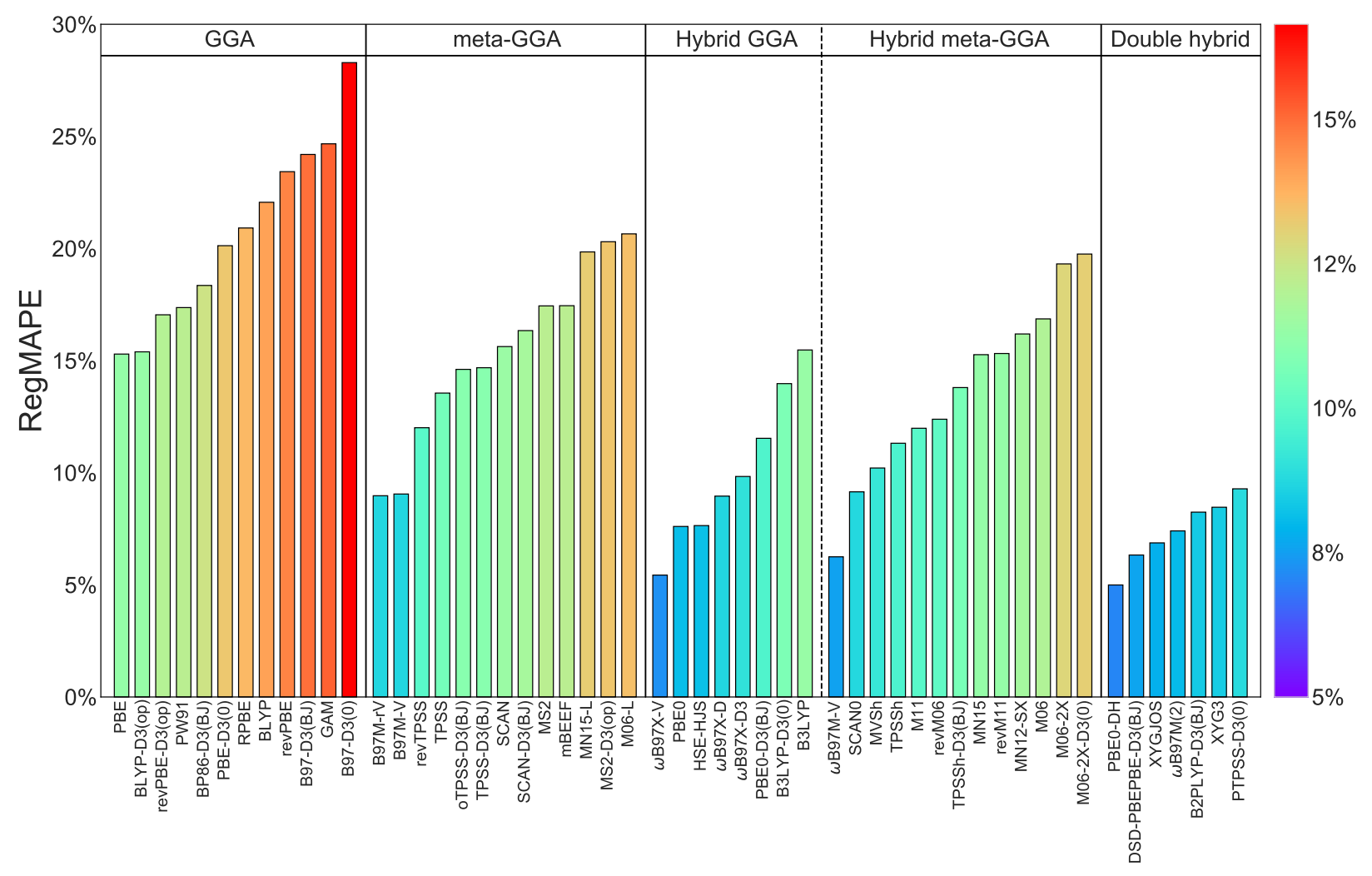

Figure 2: Performance of density functional approximations for the H2Bind $78 \times 7$ dataset assessed using regularized mean absolute percentage error (RegMAPE). The LDA density functionals, SPW92 and SVWN5, are not included in this figure and show a large RegMAPE of $63.0 \%$. 
should be given larger weight than the non-equilibrium ones.

The regularized mean absolute percentage error (RegMAPE) was formulated in Ref. 21 in order to satisfy requirement (1). RegMAPE uses percentage error in the -15 to $-25 \mathrm{~kJ} / \mathrm{mol}$ range, regularized percentage error (in order to avoid small denominators) for interaction energies weaker than $-15 \mathrm{~kJ} / \mathrm{mol}$, and absolute error for interaction energies stronger than $-25 \mathrm{~kJ} / \mathrm{mol}$. The error metrics in neighboring ranges are also smoothly interpolated. For the same amount of error, as percentage error is much larger in magnitude than absolute error, the RegMAPE error metric is able to satisfy criterion (1). For example, an error of $5 \mathrm{~kJ} / \mathrm{mol}$ for a reference interaction energy of $100 \mathrm{~kJ} / \mathrm{mol}$ will contribute 5 units to the total error while the same error for a reference interaction energy of $20 \mathrm{~kJ} / \mathrm{mol}$ will contribute 25 units to the total error. For a given binding motif, the equilibrium geometry should be given more weight as it represents the $\mathrm{H}_{2}$ interaction with the primary binding site: the main lever to tune while designing binding sites. As non-equilibrium geometries are higher in energy, they would be encountered less frequently in a molecular dynamics or Monte Carlo simulation. Hence, lower weight for non-equilibrium geometries is achieved by using the equilibrium regularization value for non-equilibrium geometries as well. As the equilibrium geometry always has a stronger interaction energy, the regularized value of its reference interaction energy, $\tilde{E}\left(r_{\text {eq }}\right)$, will be larger in magnitude in comparison to the regularized values of nonequilibrium interaction energies $\left(\tilde{E}\left(\alpha r_{\mathrm{eq}}\right), \alpha \neq 1.0\right)$. The large magnitude of the denominator will give a smaller weight to the errors of non-equilibrium geometries in comparison to the equilibrium one. RegMAPE for equilibrium and non-equilibrium geometries as defined in Eq. (4) satisfies requirement (2).

$$
\Delta E\left(\alpha r_{\mathrm{eq}}\right)=\frac{E^{\mathrm{DFA}}\left(\alpha r_{\mathrm{eq}}\right)-E^{\mathrm{ref}}\left(\alpha r_{\mathrm{eq}}\right)}{\tilde{E}\left(r_{\mathrm{eq}}\right)}, \quad \alpha \in\{0.75,0.9,1.0,1.1,1.25,1.5\}
$$

where $\Delta E\left(\alpha r_{\text {eq }}\right)$ is the RegMAPE, $E^{\mathrm{DFA}}\left(\alpha r_{\text {eq }}\right)$ and $E^{\mathrm{ref}}\left(\alpha r_{\text {eq }}\right)$ are the DFA and reference interaction energies at $\alpha r_{\text {eq }}$ geometry, and $\tilde{E}\left(r_{\text {eq }}\right)$ is the regularized interaction energy for 
the equilibrium geometry. As the vertical interaction energy is located at the minimum of the PEC, the error in this data point is regularized using the vertical reference interaction energy.

The performance of DFAs assessed by the RegMAPE error metric is shown in Fig. 2 and Table (3). While there are some similarities in the relative ordering of density functionals for RegMAPE and RMSE error metrics, there are also noteworthy differences. Again, PBE0-DH shows the best performance with the least RegMAPE of 5.0\%. It is followed by the $\omega$ B97X-V and $\omega \mathrm{B} 97 \mathrm{M}-\mathrm{V}$ density functionals which have RegMAPEs of $5.4 \%$ and $6.3 \%$ respectively. The DSD-PBEPBE-D3(BJ) density functional, which was the best performing density functional in the H2Bind275 dataset with RegMAPE of $4.9 \%$, is the fourth best performing DFA for the H2Bind $78 \times 7$ dataset. The small decline in the performance of DSD-PBEPBE-D3(BJ) can be attributed to its poor performance for the non-equilibrium geometries as shown in Table S2. Another noteworthy decline in performance is that of the B97M-V and B97M-rV functionals. These functionals were the best performing semi-local density functionals in the H2Bind275 dataset (ranked $5^{\text {th }}$ and $6^{\text {th }}$ ), and were recommended as inexpensive alternatives to the best performing and expensive density functionals. ${ }^{21}$ However, their performance in the current H2Bind $78 \times 7$ dataset deteriorates with B97M-rV and B97M-V yielding errors of $9.0 \%$ and $9.1 \%$ (ranked $14^{\text {th }}$ and $15^{\text {th }}$ ) respectively. While this reflects their lacklustre performance for the non-equilibrium geometries, they still remain the best performing semilocal functionals. The next best performing semi-local density functional, revTPSS, is ranked $21^{\text {st }}$ and shows a RegMAPE of $12.0 \%$. The performance of PBE0 and B2PLYP-D3(BJ) DFAs shows significant improvement relative to their performance in the H2Bind275 dataset with both density functionals entering the top 10 category for the H2Bind $78 \times 7$ dataset.

Other general trends also hold for DFAs assessed with the RegMAPE error metric. The best DFA of each rung of the Jacob's ladder outperforms the best DFA from the rung below it. The best performing meta-GGA functional is B97M-rV with a RegMAPE of $9.0 \%$ and the best GGA is PBE with a RegMAPE of $15.3 \%$. The effect of addition of 


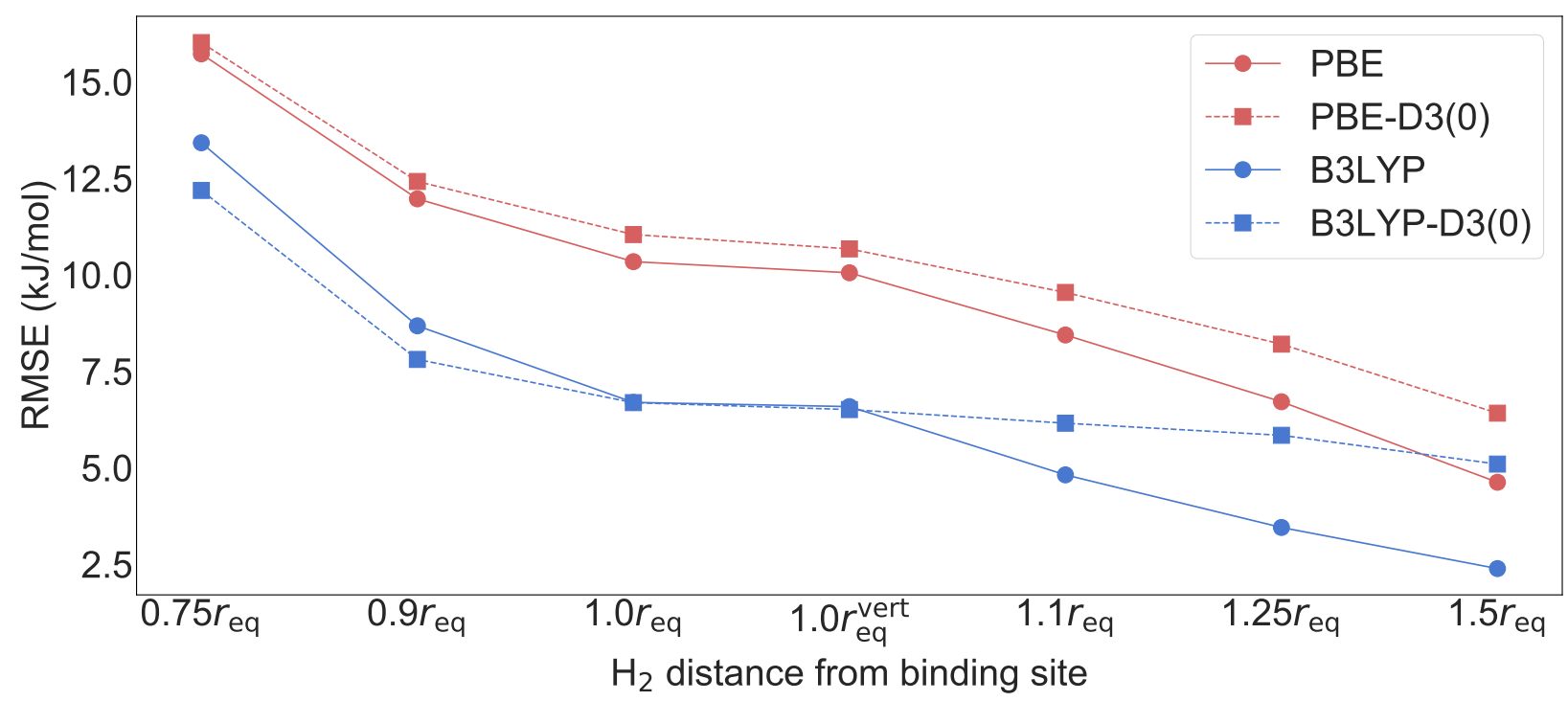

Figure 3: Effect of addition of empirical dispersion corrections on the RMSE of overbinding (PBE) and underbinding (B3LYP) density functionals at different points on the potential energy curve.

empirical dispersion correction can also be assessed using the mean signed error (MSE) and RegMAPE metrics. Addition of dispersion correction improves the performance only if the parent density functional has a systematic underbinding problem (characterized by a positive value of MSE). For example, B3LYP has an MSE of $2.7 \mathrm{~kJ} / \mathrm{mol}$ and a RegMAPE of $15.5 \%$ and is systematically underbinding $\mathrm{H}_{2}(\mathrm{~s})$. Addition of a dispersion correction to B3LYP leads to the B3LYP-D3(0) functional which overcomes this underbinding problem. B3LYPD3(0) slightly overbinds with an MSE of $-0.8 \mathrm{~kJ} / \mathrm{mol}$, but shows an improved RegMAPE of 14.0\%. Addition of DFT-D corrections also improves the performance of other underbinding DFAs like revPBE and BLYP. However, addition of these corrections to parent DFAs that are already overbinding exacerbates the overbinding issue leading to poorer performance as exemplified by PBE, PBE0, TPSS, SCAN, and MS2 functionals. Remarkably, PBE without any dispersion correction is the best performing GGA. As empirical dispersion correction is distance dependent, it is interesting to see its effect at different points on the PEC. For overbinding functionals, addition of dispersion correction worsens their performance across the PEC as exemplified by the PEC of PBE and PBE-D3(0) in Fig. 3. The difference between 
the RMSEs of PBE and PBE-D3(0) increases with increasing distance of $\mathrm{H}_{2}$ with the binding site as dispersion corrections are usually damped in the short range. Dispersion corrections improve the performance of underbinding functionals in the short range. However, in the long range, dispersion corrections overestimate its magnitude, causing BLYP, B3LYP, and revPBE to overbind in the elongated regime. MSEs and RMSEs of all the DFAs containing dispersion corrections and their corresponding parent functionals is shown in Table S3.

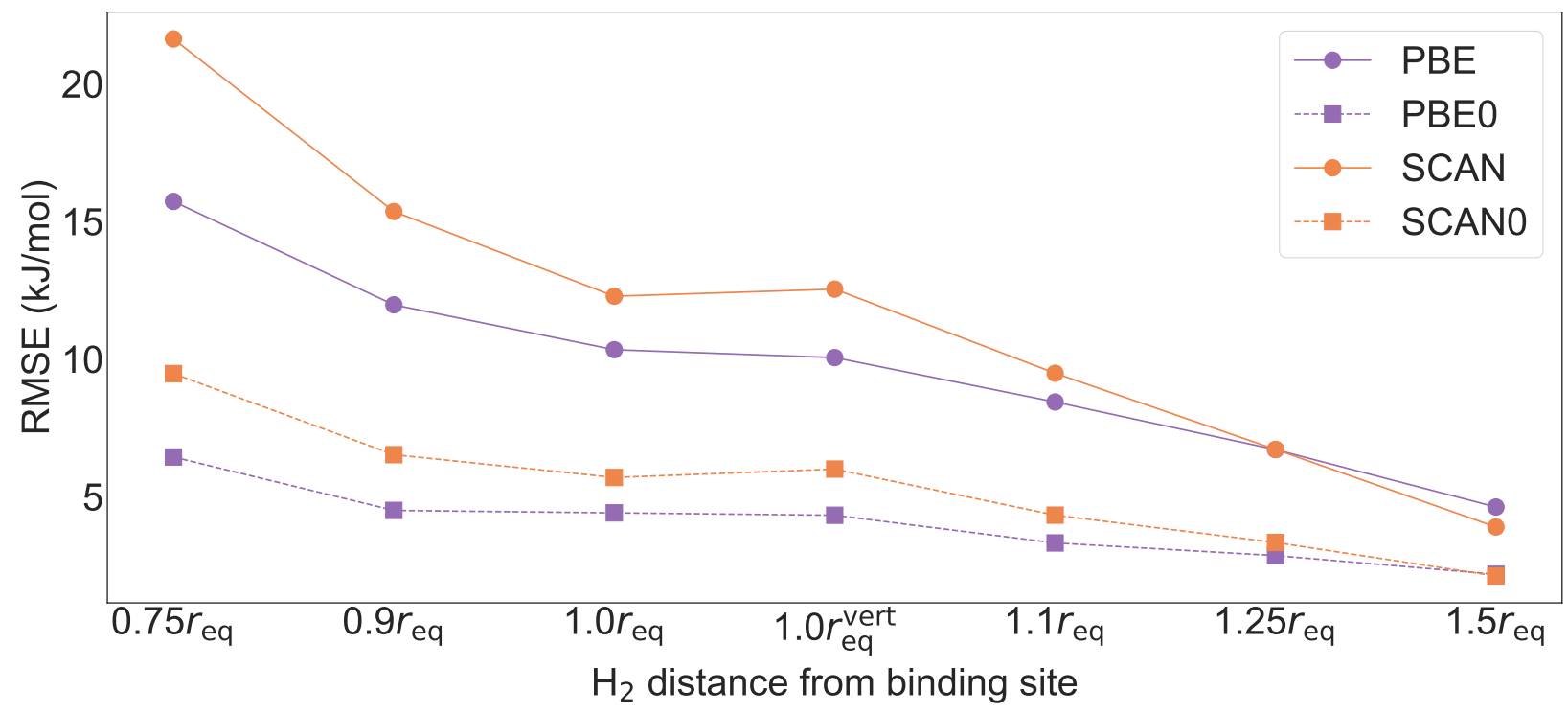

Figure 4: Performance of density functionals of the same family with and without Hartree Fock exchange at different points on the potential energy curve.

Addition of $\mathrm{HF}$ exact exchange is essential to ameliorate the effect of self interaction error in density functionals. Comparing DFAs belonging to the same family, addition of HF exchange improves the performance of semi-local functionals for the H2Bind $78 \times 7$ dataset. PBE0, which contains $25 \% \mathrm{HF}$ exchange, is ranked the $7^{\text {th }}$ with a RegMAPE of $7.6 \%$. In contrast, the PBE functional is ranked $29^{\text {th }}$ with a RegMAPE for $15.3 \%$, more than two times that of PBE0. HF exchange is a short-range effect and addition of HF exchange improves the performance of density functionals in the short range as shown in Fig. 4. While the hybrid functional performs better than its semi-local counterpart throughout the PEC, its effect is more pronounced in the compressed region than the elongated region. The SCAN and SCAN0 functionals show RMSEs of 21.6 and $9.5 \mathrm{~kJ} / \mathrm{mol}$ (a difference of $12.2 \mathrm{~kJ} / \mathrm{mol}$ ) at 
$0.75 r_{\text {eq }}$ of the PEC. Their RMSEs at $1.5 r_{\text {eq }}$ is 3.9 and $2.1 \mathrm{~kJ} / \mathrm{mol}$, with the hybrid functional improving on the semi-local one by only $1.8 \mathrm{~kJ} / \mathrm{mol}$.

The RegMAPE error metric gives larger weights to data points whose reference interaction energies are in the interesting range for $\mathrm{H}_{2}$ storage. Further, it gives more weight to the equilibrium data point than non-equilibrium data points. The relative weights of data points on the PES can be further tuned in order to assess the origin of errors of different DFAs. Elongated geometries are encountered more often than compressed geometries in porous material capable of storing $\mathrm{H}_{2}$. Compressed geometries are also much higher in energy (geometries compressed by $25 \%$ are almost always repulsive) and are encountered less often in simulations. This would suggest retuning the weights of the regularized errors by giving larger weights to equilibrium and elongated regions of the PEC. The weighted RegMAPE (denoted as wRegMAPE or $\Delta E_{w}$ ) is defined as:

$$
\Delta E_{w}=\sum_{i} \frac{w_{i} \Delta E\left(\alpha_{i} r_{\mathrm{eq}}\right)}{7} \quad \text { s.t. } \sum_{i} w_{i}=7
$$

where $\Delta E\left(\alpha_{i} r_{\text {eq }}\right)$ is the RegMAPE at the point $\alpha_{i} r_{\text {eq }}$ defined in Eq. (4). Ensuring that the weights sum up to 7 would enable an apples-to-apples comparison of wRegMAPE and RegMAPE. In the case of RegMAPE, $w_{i}=1$, for all values of $i$. Reducing the weights of the compressed geometries with the scheme shown in Table 4, the wRegMAPE can be computed using Eq. (5). This wRegMAPE metric, shown in Table 5, gives more weight to the elongated geometries. As the vertical interaction is computed its respective PEC minimum, the $1.0 r_{\mathrm{eq}}^{\mathrm{ver}}$ data point is assigned a weight equal to that of the adiabatic interaction energy at PEC minimum.

Table 4: Weights for different points on the adiabatic PEC and vertical interaction energy for calculating the weighted regularized mean absolute percentage error (wRegMAPE) metric.

\begin{tabular}{|c|ccccccc|}
\hline PEC location & $0.75 r_{\text {eq }}$ & $0.9 r_{\mathrm{eq}}$ & $1.0 r_{\mathrm{eq}}$ & $1.0 r_{\mathrm{eq}}^{\mathrm{vert}}$ & $1.1 r_{\mathrm{eq}}$ & $1.25 r_{\mathrm{eq}}$ & $1.5 r_{\mathrm{eq}}$ \\
\hline Weight $\left(w_{i}\right)$ & 0.75 & 0.91 & 1.07 & 1.07 & 1.07 & 1.07 & 1.07 \\
\hline
\end{tabular}

Comparing the magnitude of the wRegMAPE (Table 5) of different DFAs to their cor- 
Table 5: Weighted regularized mean absolute percentage error (wRegMAPE) for selected density functional approximations.

\begin{tabular}{ccc}
\hline Rank & DFA & wRegMAPE (\%) \\
\hline 1 & PBE0-DH & 4.8 \\
2 & $\omega B 97 X-V$ & 5.2 \\
3 & DSD-PBEPBE-D3(BJ) & 5.9 \\
4 & $\omega B 97 M-V$ & 6.0 \\
5 & XYGJ-OS & 6.5 \\
6 & $\omega B 97 M(2)$ & 7.0 \\
7 & PBE0 & 7.4 \\
8 & HSE-HJS & 7.4 \\
9 & XYG3 & 7.9 \\
10 & B2PLYP-D3(BJ) & 8.0 \\
11 & B97M-rV & 8.4 \\
12 & B97M-V & 8.4 \\
13 & wB97X-D & 8.5 \\
14 & SCAN0 & 8.7 \\
15 & PTPSS-D3(0) & 9.1 \\
\hline
\end{tabular}

responding RegMAPE (Table 3), we can notice that the wRegMAPEs are slightly smaller. Smaller wRegMAPEs suggest that density functionals perform better for the equilibrium and elongated geometries in comparison to the compressed ones. However, the relative ordering of density functionals remains more or less the same. The top five best performing DFAs (PBE0-DH, $\omega B 97 \mathrm{X}-\mathrm{V}, \omega \mathrm{B} 97 \mathrm{M}-\mathrm{V}, \mathrm{DSD}-\mathrm{PBEPBE}-\mathrm{D} 3(\mathrm{BJ})$, and XYGJ-OS) according to the RegMAPE metric are also the five best performing functionals according to the wRegMAPE metric. We see that the recently parametrized $\omega \mathrm{B} 97 \mathrm{M}(2)$ double hybrid density functional, which uses $\omega$ B97M-V orbitals, is ranked sixth with wRegMAPE of $7.0 \%$.

\section{Performance of Density Functional Approximation for Geometries}

All the equilibrium geometries for this dataset were obtained by geometry optimization using the $\omega$ B97M-V density functional in the def2-TZVPD basis set. ${ }^{92}$ With the exception of two chemical systems $\left(\mathrm{AlF}_{3}-\mathrm{H}_{2}\right.$ and $\left.\mathrm{Ti}^{+}-\left(\mathrm{H}_{2}\right)_{2}\right)$, the $\mathrm{CCSD}(\mathrm{T}) / \mathrm{CBS}$ equilibrium geometry of all other chemical systems coincides (up to sampling precision) with the $\omega$ B97M-V/def2TZVPD equilibrium geometry. This further validates the choice of equilibrium geometries 


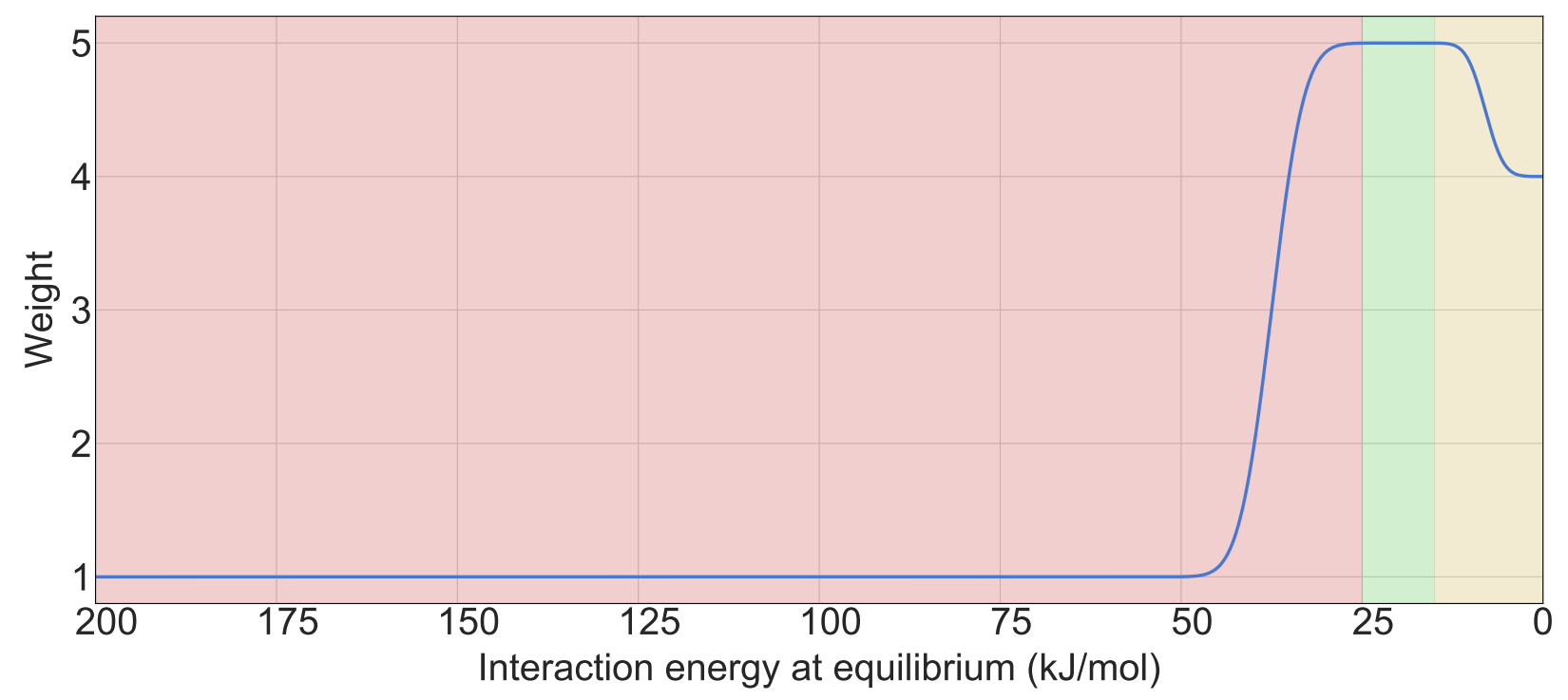

Figure 5: Weights of different chemical species as a function of their reference adiabatic interaction energy at equilibrium.

for the H2Bind $78 \times 7$ dataset.

In order to assess the error in prediction of equilibrium geometry in a manner that is sensitive for $\mathrm{H}_{2}$ storage purposes, we have devised a weighting scheme that gives larger weights to more relevant data points. Data points with reference adiabatic interaction energy at equilibrium $\left(E^{\mathrm{ref}}\left(1.0 r_{\mathrm{eq}}\right)\right)$ in the range of -15 to $-25 \mathrm{~kJ} / \mathrm{mol}$ are given a weight of 5.0. Equilibrium interaction energies stronger than $-25 \mathrm{~kJ} / \mathrm{mol}$ are assigned a weight of 1.0. These weights were chosen to reflect the relative importance of these data points in the RegMAPE metric. In the RegMAPE metric, a density functional yielding an error of 1 $\mathrm{kJ} / \mathrm{mol}$ in the strong binding regime contributes 1 unit to the total error as absolute error metric is used in this regime. A DFA with an error of the $1 \mathrm{~kJ} / \mathrm{mol}$ in the middle of the favorable regime for $\mathrm{H}_{2}$ storage (that is at $-20 \mathrm{~kJ} / \mathrm{mol}$ ) contributes 5 units to the total error as percentage error metric is used. The RegMAPE metric assigns a weight that is 5 times larger to the species in the favorable regime in comparison to the strong binders, thus justifying the weights of 5.0 and 1.0 in Fig. (5). The weak binders with equilibrium interaction energies weaker than $-15 \mathrm{~kJ} / \mathrm{mol}$ are mostly comprised of the organic ligands. These species are ubiquitously found in porous materials capable of adsorbing $\mathrm{H}_{2}$ (like MOFs) 
and form secondary binding sites for $\mathrm{H}_{2}$. As this regime is not as important as the favorable one, it is assigned a weight of 4.0. This weighting scheme is used to form the weighted mean signed error (wMSE) and weighted mean unsigned error (wMAE) metrics in Fig. (5). The reference adiabatic interaction energy at equilibrium decides the weight of the corresponding PEC. As the vertical interaction energy does not lie on the adiabatic PEC, those data points were not included in the analyses in this section.

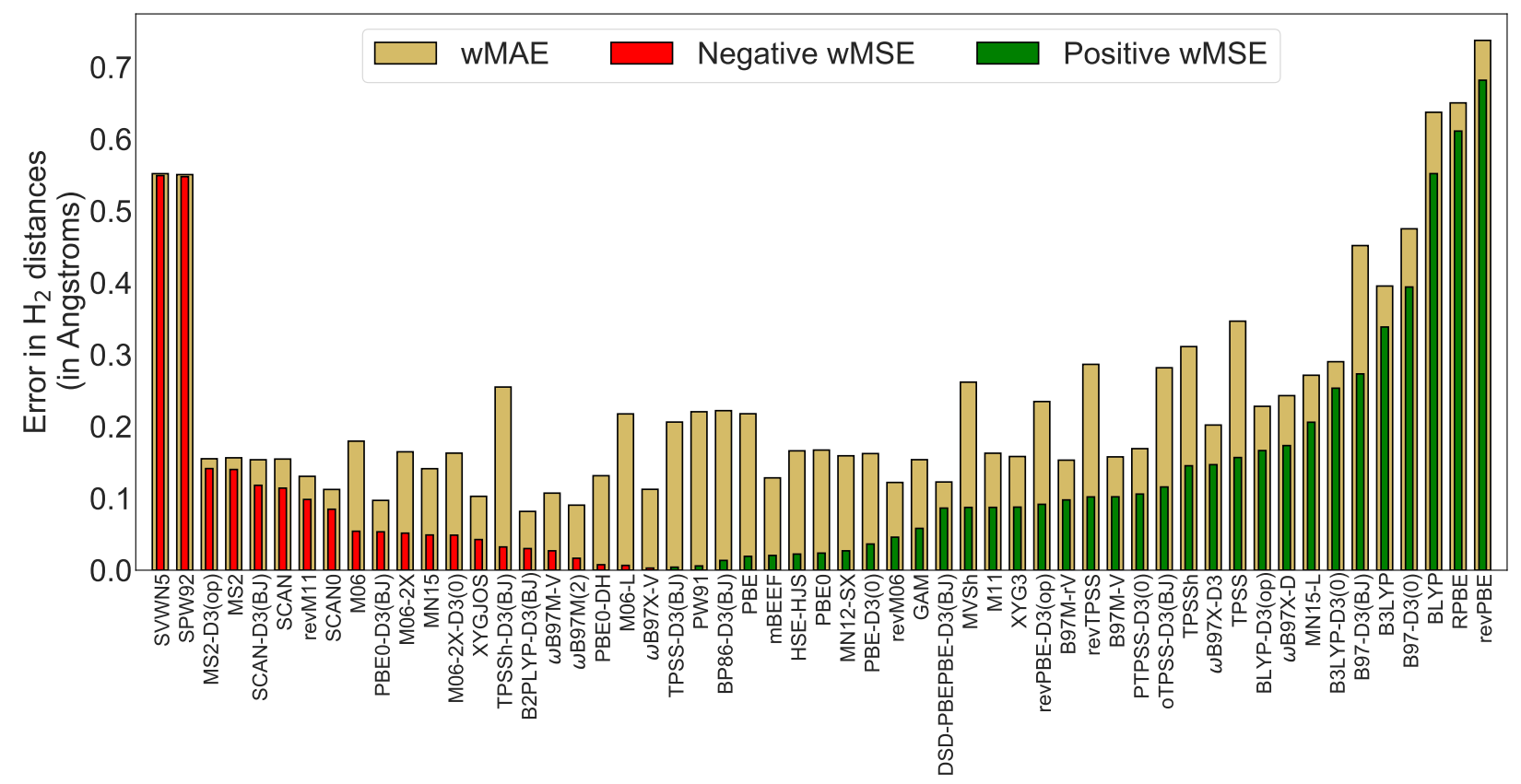

Figure 6: Weighted mean absolute error (wMAE) and weighted mean signed error (wMSE) of equilibrium $\mathrm{H}_{2}$ distances predicted by different DFAs.

Most DFAs predict longer equilibrium binding motif $-\mathrm{H}_{2}$ distances which is shown as a positive value of wMSE in Fig. 6. With the exception of the LDA density functionals, we see that all other density functionals which predict shorter equilibrium $\mathrm{H}_{2}$ distances have a very small negative wMSE $(>-0.15 \AA)$. The double hybrid density functional B2PLYP-D3(BJ) gives the best performance for predicting equilibrium geometry with a wMAE of $0.08 \AA$. It is closely followed by the recently parametrized double hybrid $\omega \mathrm{B} 97 \mathrm{M}(2)$ density functional with a wMAE of $0.09 \AA$. Both of these DFAs perform much better for equilibrium geometries in comparison to their performance for PECs. It is also rather surprising to note the less 
good performance of PBE0-DH (wMAE of $0.13 \AA$ ), which is the best performing DFA for binding energies according to RMSE and RegMAPE. On the other hand, PBE0-D3(BJ) gives good geometries (ranked $3^{\text {rd }}$ with wMAE of $0.10 \AA$ ) but its performance for PECs is mediocre (ranked $19^{\text {th }}$ with a RegMAPE of $11.5 \%$ ). However, other top performing DFAs in the energetics category like XYGJ-OS, $\omega \mathrm{B} 97 \mathrm{M}-\mathrm{V}$, and $\omega \mathrm{B} 97 \mathrm{X}-\mathrm{V}$ also perform well for geometries giving low wMAEs of $0.10 \AA, 0.11 \AA$, and $0.11 \AA$ respectively. In particular, $\omega \mathrm{B} 97 \mathrm{X}-\mathrm{V}$ shows no systematic error with virtually zero wMSE. It is also interesting to note the good performance of some semi-local density functionals like mBEEF and B97M-rV which give very low errors despite having no HF exchange. In light of these observations, and given the enhanced computational cost of double hybrid DFA nuclear gradients, ${ }^{97}$ one can use hybrid DFAs like PBE0-D3(BJ) or $\omega$ B97M-V to perform a geometry optimization and then use the optimized geometry to perform a single point interaction energy calculation using a hybrid or double hybrid functional.

Another noticeable tread is the performance of DFAs upon the addition of some form of empirical dispersion correction. Addition of empirical dispersion corrections to DFAs reduces their errors for equilibrium $\mathrm{H}_{2}$ distance prediction when the parent functional overestimates it. For example, the addition of the D3(op) correction to revPBE decreases its wMSE from $0.68 \AA$ to $0.09 \AA$ (concurrently decreasing wMAE from $0.74 \AA$ to $0.23 \AA$ ). The performance of the commonly used density functional B3LYP and M06-2X is quite poor with large wMSE and wMAEs.

Typically, DFAs are used to optimize geometries of complexes containing an $\mathrm{H}_{2}$ bound to a binding motif. After the geometry optimization has converged to a minimum on the potential energy surface, the binding energy of $\mathrm{H}_{2}$ is computed as the difference between the energy of the complex at the minimum of the potential energy surface and energies of the binding motif and $\mathrm{H}_{2}$ in isolation. Alternatively, DFAs can also be used in molecular dynamics and Monte Carlo simulations either directly ${ }^{98}$ or indirectly (as reference energies for parametrizing force fields). ${ }^{99-102}$ In these typical use cases, error in equilibrium binding 
Table 6: Performance of density functional approximations (DFAs) for predicting $\mathrm{H}_{2}$ binding energy at equilibrium geometry. The adiabatic regularized mean absolute percentage error $\left(\right.$ RegMAPE $_{\text {ad }}$ ) for 15 best performing DFAs and some commonly used DFAs are shown.

\begin{tabular}{ccc}
\hline Rank & DFA & RegMAPE ${ }_{\mathrm{ad}}$ \\
\hline 1 & $\omega$ B97X-V & 4.7 \\
2 & DSD-PBEPBE-D3(BJ) & 4.7 \\
3 & PBE0-DH & 5.3 \\
4 & wB97M-V & 6.0 \\
5 & XYGJ-OS & 7.1 \\
6 & B97M-rV & 7.2 \\
7 & B97M-V & 7.2 \\
8 & $\omega$ B97M(2) & 7.4 \\
9 & $\omega B 97 X-D$ & 7.8 \\
10 & XYG3 & 7.9 \\
11 & B2PLYP-D3(BJ) & 8.3 \\
12 & PBE0 & 8.4 \\
13 & HSE-HJS & 8.4 \\
14 & WB97X-D3 & 9.5 \\
15 & SCAN0 & 9.7 \\
28 & B3LYP & 15.7 \\
30 & PBE & 16.3 \\
31 & SCAN & 16.4 \\
33 & revPBE-D3(op) & 17.3 \\
42 & mBEEF & 19.9 \\
48 & M06-2X & 22.3 \\
51 & B97-D3(BJ) & 24.4 \\
52 & GAM & 26.5 \\
53 & B97-D3(0) & 29.4 \\
\hline
\end{tabular}


energy can be attributed to two sources: (1) Inaccurate prediction of equilibrium geometry (2) Incorrect prediction of binding energy for the equilibrium geometry. Using DFAs for modeling $\mathrm{H}_{2}$ binding materials, the DFA equilibrium geometry is typically used for computing the equilibrium binding energy.

We can assess the effect of relaxing the geometry along the PEC (defined by each DFA), the interaction coordinate of $\mathrm{H}_{2}$ with the binding site. Geometry optimization along this coordinate can either improve or deteriorate the performance of density functionals. The performance of selected density functionals for predicting the minimum energy geometry on its $\mathrm{PEC}$ and the $\mathrm{H}_{2}$ binding energy for this geometry assessed by the RegMAPE error metric is shown in Table 6 (the complete table showing the performance of all 55 DFAs is included in Table S4).

As both the reference and DFA geometries are relaxed along the potential energy curve, this error metric is adiabatic (ad) in nature as reflected in its subscript (RegMAPE $\mathrm{ad}_{\mathrm{ad}}$ ). The top five density functionals by the RegMAPE $\mathrm{ad}_{\mathrm{d}}$ error metric are also the top five best performers according to their RegMAPE errors (Table 3), further emphasizing the superior performance of these DFAs for computing $\mathrm{H}_{2}$ interaction energies. While the top five density functionals remain the same, it is interesting to note small changes in their order. $\omega$ B97X$\mathrm{V}$ is the best performing DFA with a RegMAPE ad of $4.65 \%$ which is very closely followed by DSD-PBEPBE-D3(BJ) with a RegMAPE $\mathrm{ad}_{\mathrm{ad}}$ of $4.68 \%$. Another noteworthy difference is the performance of the B97M-rV and the B97M-V functionals which are ranked sixth and seventh with $\operatorname{RegMAPE}_{\mathrm{ad}}$ of $7.22 \%$ and $7.23 \%$ respectively. These DFAs were ranked $12^{\text {th }}$ and $13^{\text {th }}$ with RegMAPE of $9.0 \%$ and $9.1 \%$. These functionals show a favorable cancellation of error in prediction of $\mathrm{H}_{2}$ equilibrium binding energies when the equilibrium geometry is also optimized using the same functional. As these density functionals also do not have any HF exchange, they are computationally less expensive making them well-suited for applications in high-throughput material screening. The rVV10 non-local functional, which is an approximation ${ }^{65}$ of the VV10 non-local functional, also allows for efficient evaluation 
in a plane wave framework and can be useful for modeling periodic systems like MOFs. A thorough assessment of the DFA geometry relaxed on the entire potential energy surface, not just along the one-dimensional PEC, is beyond the scope of this work and we refer interested readers to Ref. 28 for a detailed discussion of this topic.

\section{Conclusions}

The H2Bind275 dataset published recently ${ }^{21}$ assesses the performance of density functionals for predicting the interaction energy of $\mathrm{H}_{2}$ with different model binding motifs at the equilibrium geometry. In this work, we have assessed the ability of DFAs to predict $\mathrm{H}_{2}$ interaction energies with binding motifs accurately throughout the PEC, not just at equilibrium geometry. To that end, we have extended our previous H2Bind275 dataset by adding two compressed and three elongated geometries along the PEC to form the H2Bind $78 \times 7$ dataset. The H2Bind $78 \times 7$ dataset comprises 545 data points at different fixed points along 78 PECs of various model binding motifs with $\mathrm{H}_{2}$. Reference interaction energies for all data points were computed using $\operatorname{CCSD}(\mathrm{T})$ extrapolated to the complete basis set limit. The performance of 55 DFAs was assessed with the CCSD $(\mathrm{T})$ reference interaction energies using multiple error metrics. The RMSE metric is democratic and gives equal important to all the 545 data points. The RegMAPE metric, on the other hand, gives more weight to binding motifs with interaction energies in the range of -15 to $-25 \mathrm{~kJ} / \mathrm{mol}$ at equilibrium geometry. For each binding motif, the RegMAPE metric is designed to give more weight to the equilibrium than non-equilibrium data points as the latter are encountered less often in modeling and simulation. DFAs are also assessed on the basis of their predicted equilibrium geometry and binding energy at predicted equilibrium geometry.

The CCSD $(\mathrm{T})$ reference interaction energies for the $\mathrm{H} 2 \mathrm{Bind} 78 \times 7$ dataset span a wide range of attractive and repulsive interaction energies. As repulsive geometries are usually not included in non-covalent interaction energy datasets, the H2Bind $78 \times 7$ dataset adds 
considerably to the diversity of the available datasets. The non-empirical double hybrid functional, PBE0-DH, shows the least error (RMSE of $2.9 \mathrm{~kJ} / \mathrm{mol}$ and RegMAPE of 5.0\%) in predicting $\mathrm{H}_{2}$ binding energy throughout the PEC. The $\omega \mathrm{B} 97 \mathrm{X}-\mathrm{V}, \omega \mathrm{B} 97 \mathrm{M}-\mathrm{V}$, and DSDPBEPBE-D3(BJ) density functionals are also top performers. The semi-local density functionals, B97M-V and B97M-rV, show poorer performance for the H2Bind78 $\times 7$ dataset, in comparison to the previous H2Bind275 dataset using the RegMAPE error metric. For the H2Bind $78 \times 7$ dataset, B97M-V and B97M-rV are ranked $13^{\text {th }}$ and $12^{\text {th }}$ respectively with RegMAPEs of $9.1 \%$ and $9.0 \%$. Previously in the H2Bind275 dataset, they were ranked $5^{\text {th }}$ and $6^{\text {th }}$ with RegMAPE of $6.8 \%$. In general, the good performance of the top density functionals in the H2Bind275 dataset continues for the H2Bind $78 \times 7$ dataset. Addition of DFT-D empirical dispersion correction increases the accuracy of underbinding density functionals like revPBE, BLYP, and B3LYP. This addition also decreases the accuracy of overbinding parent density functionals like PBE, PBE0, TPSS, SCAN, and MS2. As DFTD empirical dispersion corrections are distance dependent, their effect is not felt uniformly across the PEC. The effect of addition of HF exchange, a short-ranged effect, improves the performance of density functionals in the compressed regime more than in the elongated regime of the PEC, thus playing a crucial role in accurately predicting the repulsive wall of the PEC. The weighted RegMAPE metric gives smaller weights to DFA errors in the compressed region of the PEC. This metric shows that, in general, DFAs perform better in the equilibrium and elongated regime than in the compressed region.

Assessment of DFAs for predicting equilibrium geometries reveals that PBE0-DH, which is the best performer for energies, shows less good performance for geometries. However, other hybrid functionals like $\omega \mathrm{B} 97 \mathrm{M}-\mathrm{V}$ and $\omega \mathrm{B} 97 \mathrm{X}-\mathrm{V}$ give good performance for both geometries and energies. Using the adiabatic RegMAPE metric $\left(\operatorname{RegMAPE}_{\mathrm{ad}}\right)$ reveals that the semi-local DFAs, B97M-V and B97M-rV, show very small errors. They benefit significantly from cancellation between geometry-driven and energy-driven errors. $\omega \mathrm{B} 97 \mathrm{M}-\mathrm{V}$ and $\omega \mathrm{B} 97 \mathrm{X}-$ $\mathrm{V}$ are the only density functionals that are not double hybrids which consistently show good 
performance for all the error metrics (energy and geometry-related) defined in this work. As these hybrid functionals have significantly lower computational cost in comparison to double hybrids, we recommend their usage for $\mathrm{H}_{2}$ binding applications.

The H2Bind $78 \times 7$ dataset, consisting of highly accurate reference interaction energies, represents a distinctive addition to other non-covalent interaction energy databases. More than half of this dataset consists of transition metal species which are usually underrepresented in non-covalent interaction energy datasets. This dataset is composed of complete PECs, rather than just PEC minimum geometries - only a handful of the non-covalent interaction energy datasets contain this information. Besides, almost all of the PECs in this dataset sample the repulsive wall. For these reasons, using the H2Bind $78 \times 7$ dataset in training or validating DFAs can improve their performance and transferability. This work further validates the selection of best performing density functionals for the H2Bind275 dataset using a semi-independent dataset that is about two times larger. The definition and gener-

alization of different error metrics (RegMAPE, wRegMAPE, and RegMAPE ${ }_{a d}$ ) can be used for assessment of other similar datasets with well-defined schemes for weighting different data points. As force field parametrization requires good reference energies throughout the potential energy surface, the top performing density functionals in this work can be used for generating them.

\section{Acknowledgement}

This work was supported by the Hydrogen Materials - Advanced Research Consortium (HyMARC), established as part of the Energy Materials Network under the U.S. Department of Energy, Office of Energy Efficiency and Renewable Energy, under Contract No. DE-AC0205CH11231. The following author declares a competing financial interest. M. H. G. is a part owner of Q-Chem, Inc. 


\section{Supporting Information Available}

Additional information regarding the parameters used for double hybrid density function-

als, performance of density functionals for the non-equilibrium subset of the $\mathrm{H} 2 \mathrm{Bind} 78 \times 7$

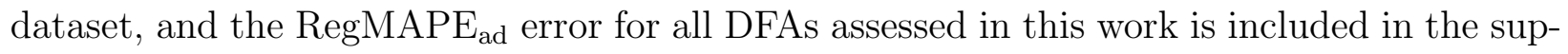
plementary information. CCSD $(\mathrm{T}) / \mathrm{CBS}$ and DFA interaction energies for all 55 functionals are provided in the file supporting_information.xlsx. The geometries of all complexes in the H2Bind78 $\times 7$ are included in geometries.zip file.

\section{References}

(1) Takagi, H.; Hatori, H.; Soneda, Y.; Yoshizawa, N.; Yamada, Y. Adsorptive hydrogen storage in carbon and porous materials. Mater. Sci. Eng., B 2004, 108, 143-147.

(2) Thomas, K. M. Hydrogen adsorption and storage on porous materials. Catal. Today 2007, 120, 389-398.

(3) Murray, L. J.; Dincă, M.; Long, J. R. Hydrogen storage in metal-organic frameworks. Chem. Soc. Rev. 2009, 38, 1294-1314.

(4) Park, N.; Choi, K.; Hwang, J.; Kim, D. W.; Kim, D. O.; Ihm, J. Progress on firstprinciples-based materials design for hydrogen storage. Proc. Natl. Acad. Sci. U. S. A. 2012, 109, 19893-19899.

(5) Allendorf, M. D.; Hulvey, Z.; Gennett, T.; Ahmed, A.; Autrey, T.; Camp, J.; Seon Cho, E.; Furukawa, H.; Haranczyk, M.; Head-Gordon, M.; Jeong, S.; Karkamkar, A.; Liu, D. J.; Long, J. R.; Meihaus, K. R.; Nayyar, I. H.; Nazarov, R.; Siegel, D. J.; Stavila, V.; Urban, J. J.; Veccham, S. P.; Wood, B. C. An assessment of strategies for the development of solid-state adsorbents for vehicular hydrogen storage. Energy Environ. Sci. 2018, 11, 2784-2812. 
(6) Colon, Y. J.; Fairen-Jimenez, D.; Wilmer, C. E.; Snurr, R. Q. High-throughput screening of porous crystalline materials for hydrogen storage capacity near room temperature. J. Phys. Chem. C 2014, 118, 5383-5389.

(7) Thornton, A. W.; Simon, C. M.; Kim, J.; Kwon, O.; Deeg, K. S.; Konstas, K.; Pas, S. J.; Hill, M. R.; Winkler, D. A.; Haranczyk, M.; Smit, B. Materials Genome in Action: Identifying the Performance Limits of Physical Hydrogen Storage. Chem. Mater. 2017, 29, 2844-2854.

(8) Ahmed, A.; Seth, S.; Purewal, J.; Wong-Foy, A. G.; Veenstra, M.; Matzger, A. J.; Siegel, D. J. Exceptional hydrogen storage achieved by screening nearly half a million metal-organic frameworks. Nat. Commun. 2019, 10, 1-9.

(9) Kapelewski, M. T.; Geier, S. J.; Hudson, M. R.; Stck, D.; Mason, J. A.; Nelson, J. N.; Xiao, D. J.; Hulvey, Z.; Gilmour, E.; FitzGerald, S. A.; Head-Gordon, M.; Brown, C. M.; Long, J. R. M2(m-dobdc) (M = Mg, Mn, Fe, Co, Ni) MetalOrganic Frameworks Exhibiting Increased Charge Density and Enhanced H2 Binding at the Open Metal Sites. J. Am. Chem. Soc. 2014, 136, 12119-12129.

(10) Tsivion, E.; Veccham, S. P.; Head-Gordon, M. High-Temperature Hydrogen Storage of Multiple Molecules: Theoretical Insights from Metalated Catechols. ChemPhysChem 2017, 18, 184-188.

(11) Garrone, E.; Bonelli, B.; Otero Areán, C. Enthalpy-entropy correlation for hydrogen adsorption on zeolites. Chem. Phys. Lett. 2008, 456, 68-70.

(12) Bhatia, S. K.; Myers, A. L. Optimum conditions for adsorptive storage. Langmuir 2006, 22, 1688-1700.

(13) Bae, Y. S.; Snurr, R. Q. Optimal isosteric heat of adsorption for hydrogen storage and delivery using metal-organic frameworks. Microporous Mesoporous Mater. 2010, 132, 300-303. 
(14) Mueller, T.; Ceder, G. A density functional theory study of hydrogen adsorption in MOF-5. J. Phys. Chem. B 2005, 109, 17974-17983.

(15) Tsivion, E.; Long, J. R.; Head-Gordon, M. Hydrogen physisorption on metal-organic framework linkers and metalated linkers: A computational study of the factors that control binding strength. J. Am. Chem. Soc. 2014, 136, 17827-17835.

(16) Cabria, I.; López, M.; Alonso, J. Hydrogen storage capacities of nanoporous carbon calculated by density functional and Møller-Plesset methods. Phys. Rev. B 2008, 78, 075415 .

(17) Cabria, I.; López, M.; Alonso, J. Simulation of the hydrogen storage in nanoporous carbons with different pore shapes. Int. J. Hydrogen Energy 2011, 36, 10748-10759.

(18) Niaz, S.; Manzoor, T.; Islam, N.; Pandith, A. H. Theoretical investigations on C2H4Nb complex as a potential hydrogen storage system, using moller-plesset (MP2) and density functional theory. Int. J. Quantum Chem. 2014, 114, 449-457.

(19) Kocman, M.; Jurečka, P.; Dubeckỳ, M.; Otyepka, M.; Cho, Y.; Kim, K. S. Choosing a density functional for modeling adsorptive hydrogen storage: reference quantum mechanical calculations and a comparison of dispersion-corrected density functionals. Phys. Chem. Chem. Phys. 2015, 17, 6423-6432.

(20) Ma, L.-J.; Jia, J.; Wu, H.-S. Computational investigation of hydrogen storage on scandium-acetylene system. Int. J. Hydrogen Energy 2015, 40, 420-428.

(21) Veccham, S. P.; Head-Gordon, M. Density Functionals for Hydrogen Storage: Defining the H2Bind275 Test Set with Ab Initio Benchmarks and Assessment of 55 Functionals. J. Chem. Theory Comput. 2020, 16, 4963-4982.

(22) Mardirossian, N.; Head-Gordon, M. $\omega$ B97M-V: A combinatorially optimized, range- 
separated hybrid, meta-GGA density functional with VV10 nonlocal correlation. J. Chem. Phys. 2016, 144, 214110.

(23) Sillar, K.; Hofmann, A.; Sauer, J. Ab Initio Study of Hydrogen Adsorption in MOF-5. J. Am. Chem. Soc. 2009, 131, 4143-4150.

(24) Koizumi, K.; Nobusada, K.; Boero, M. Hydrogen storage mechanism and diffusion in metal-organic frameworks. Phys. Chem. Chem. Phys. 2019, 21, 7756-7764.

(25) Gráfová, L.; Pitonak, M.; Rezac, J.; Hobza, P. Comparative study of selected wave function and density functional methods for noncovalent interaction energy calculations using the extended S22 data set. J. Chem. Theory Comput. 2010, 6, 2365-2376.

(26) Jurečka, P.; Šponer, J.; Černỳ, J.; Hobza, P. Benchmark database of accurate (MP2 and CCSD (T) complete basis set limit) interaction energies of small model complexes, DNA base pairs, and amino acid pairs. Phys. Chem. Chem. Phys. 2006, 8, 1985-1993.

(27) Řezáč, J.; Riley, K. E.; Hobza, P. S66: A well-balanced database of benchmark interaction energies relevant to biomolecular structures. J. Chem. Theory Comput. 2011, 7, $2427-2438$.

(28) Witte, J.; Goldey, M.; Neaton, J. B.; Head-Gordon, M. Beyond energies: Geometries of nonbonded molecular complexes as metrics for assessing electronic structure approaches. J. Chem. Theory Comput. 2015, 11, 1481-1492.

(29) Rezac, J.; Hobza, P. Describing noncovalent interactions beyond the common approximations: How accurate is the gold standard, CCSD (T) at the complete basis set limit? J. Chem. Theory Comput. 2013, 9, 2151-2155.

(30) Raghavachari, K.; Trucks, G. W.; Pople, J. A.; Head-Gordon, M. A fifth order comparison of electron correlation theories. Chem. Phys. Lett. 1989, 157, 479-483. 
(31) Tajti, A.; Szalay, P. G.; Császár, A. G.; Kállay, M.; Gauss, J.; Valeev, E. F.; Flowers, B. A.; Vázquez, J.; Stanton, J. F. HEAT: High accuracy extrapolated ab initio thermochemistry. J. Chem. Phys. 2004, 121, 11599-11613.

(32) DeYonker, N. J.; Cundari, T. R.; Wilson, A. K. The correlation consistent composite approach (ccCA): An alternative to the Gaussian-n methods. J. Chem. Phys. 2006, 124,84108 .

(33) Karton, A.; Rabinovich, E.; Martin, J. M.; Ruscic, B. W4 theory for computational thermochemistry: In pursuit of confident sub-kJ/mol predictions. J. Chem. Phys. 2006, 125, 11599.

(34) Curtiss, L. A.; Redfern, P. C.; Raghavachari, K. Gaussian-4 theory. J. Chem. Phys. 2007, 126, 084108 .

(35) East, A. L. L.; Allen, W. D. The heat of formation of NCO. J. Chem. Phys. 1993, 99, 4638-4650.

(36) Császár, A. G.; Allen, W. D.; Schaefer, H. F. In pursuit of the ab initio limit for conformational energy prototypes. J. Chem. Phys. 1998, 108, 9751-9764.

(37) Helgaker, T.; Klopper, W.; Koch, H.; Noga, J. Basis-set convergence of correlated calculations on water. J. Chem. Phys. 1997, 106, 9639-9646.

(38) Dunning, T. H. Gaussian basis sets for use in correlated molecular calculations. I. The atoms boron through neon and hydrogen. J. Chem. Phys. 1989, 90, 1007-1023.

(39) Woon, D. E.; Dunning, T. H. Gaussian basis sets for use in correlated molecular calculations. III. The atoms aluminum through argon. J. Chem. Phys. 1993, 98, 13581371.

(40) Woon, D. E.; Dunning, T. H. Gaussian basis sets for use in correlated molecular 
calculations. V. Core-valence basis sets for boron through neon. J. Chem. Phys. 1995, 103, 4572-4585.

(41) Peterson, K. A.; Dunning, T. H. Accurate correlation consistent basis sets for molecular core-valence correlation effects: The second row atoms Al-Ar, and the first row atoms B-Ne revisited. J. Chem. Phys. 2002, 117, 10548-10560.

(42) Balabanov, N. B.; Peterson, K. A. Systematically convergent basis sets for transition metals. I. All-electron correlation consistent basis sets for the 3d elements Sc-Zn. J. Chem. Phys. 2005, 123, 64107.

(43) Mardirossian, N.; Head-Gordon, M. Thirty years of density functional theory in computational chemistry: an overview and extensive assessment of 200 density functionals. Mol. Phys. 2017, 115, 2315-2372.

(44) Perdew, J. P.; Ruzsinszky, A.; Tao, J.; Staroverov, V. N.; Scuseria, G. E.; Csonka, G. I. Prescription for the design and selection of density functional approximations: More constraint satisfaction with fewer fits. J. Chem. Phys. 2005, 123, 62201.

(45) Dirac, P. A. Note on exchange phenomena in the Thomas atom. Math. Proc. Cambridge Philos. Soc. 1930; pp 376-385.

(46) Vosko, S. H.; Wilk, L.; Nusair, M. Accurate spin-dependent electron liquid correlation energies for local spin density calculations: a critical analysis. Can. J. Phys. 1980, $58,1200-1211$.

(47) Perdew, J. P.; Wang, Y. Accurate and simple analytic representation of the electrongas correlation energy. Phys. Rev. B 1992, 45, 13244.

(48) Perdew, J.; Burke, K.; Ernzerhof, M. Generalized Gradient Approximation Made Simple. Phys. Rev. Lett. 1996, 77, 3865-3868. 
(49) Grimme, S.; Antony, J.; Ehrlich, S.; Krieg, H. A consistent and accurate ab initio parametrization of density functional dispersion correction (DFT-D) for the 94 elements H-Pu. J. Chem. Phys. 2010, 132, 154104.

(50) Hammer, B.; Hansen, L. B.; Nørskov, J. K. Improved adsorption energetics within density-functional theory using revised Perdew-Burke-Ernzerhof functionals. Phys. Rev. B 1999, 59, 7413.

(51) Zhang, Y.; Yang, W. Comment on Generalized gradient approximation made simple. Phys. Rev. Lett. 1998, 80, 890.

(52) Witte, J.; Mardirossian, N.; Neaton, J. B.; Head-Gordon, M. Assessing DFT-D3 Damping Functions Across Widely Used Density Functionals: Can We Do Better? J. Chem. Theory Comput. 2017, 13, 2043-2052.

(53) Becke, A. D. Density-functional exchange-energy approximation with correct asymptotic behavior. Phys. Rev. A 1988, 38, 3098.

(54) Lee, C.; Yang, W.; Parr, R. G. Development of the Colle-Salvetti correlation-energy formula into a functional of the electron density. Phys. Rev. B 1988, 37, 785-789.

(55) Becke, A. D. Density-functional thermochemistry. V. Systematic optimization of exchange-correlation functionals. J. Chem. Phys. 1997, 10\%, 8554-8560.

(56) Grimme, S.; Ehrlich, S.; Goerigk, L. Effect of the damping function in dispersion corrected density functional theory. J. Comput. Chem. 2011, 32, 1456-1465.

(57) Perdew, J. P.; Chevary, J. A.; Vosko, S. H.; Jackson, K. A.; Pederson, M. R.; Singh, D. J.; Fiolhais, C. Atoms, molecules, solids, and surfaces: Applications of the generalized gradient approximation for exchange and correlation. Phys. Rev. B 1992, 46, 6671-6687. 
(58) Haoyu, S. Y.; Zhang, W.; Verma, P.; He, X.; Truhlar, D. G. Nonseparable exchangecorrelation functional for molecules, including homogeneous catalysis involving transition metals. Phys. Chem. Chem. Phys. 2015, 17, 12146-12160.

(59) Tao, J.; Perdew, J. P.; Staroverov, V. N.; Scuseria, G. E. Climbing the density functional ladder: Nonempirical meta-generalized gradient approximation designed for molecules and solids. Phys. Rev. Lett. 2003, 91, 146401.

(60) Perdew, J. P.; Ruzsinszky, A.; Csonka, G. I.; Constantin, L. A.; Sun, J. Workhorse semilocal density functional for condensed matter physics and quantum chemistry. Phys. Rev. Lett. 2009, 103, 026403.

(61) Sun, J.; Ruzsinszky, A.; Perdew, J. P. Strongly constrained and appropriately normed semilocal density functional. Phys. Rev. Lett. 2015, 115, 036402.

(62) Brandenburg, J.; Bates, J.; Sun, J.; Perdew, J. Benchmark tests of a strongly constrained semilocal functional with a long-range dispersion correction. Phys. Rev. B 2016, 94, 115144.

(63) Sun, J.; Haunschild, R.; Xiao, B.; Bulik, I. W.; Scuseria, G. E.; Perdew, J. P. Semilocal and hybrid meta-generalized gradient approximations based on the understanding of the kinetic-energy-density dependence. J. Chem. Phys. 2013, 138, 044113.

(64) Mardirossian, N.; Head-Gordon, M. Mapping the genome of meta-generalized gradient approximation density functionals: The search for B97M-V. J. Chem. Phys. 2015, 142, 074111.

(65) Sabatini, R.; Gorni, T.; De Gironcoli, S. Nonlocal van der Waals density functional made simple and efficient. Phys. Rev. B 2013, 87, 4-7.

(66) Mardirossian, N.; Ruiz Pestana, L.; Womack, J. C.; Skylaris, C.-K.; Head-Gordon, T.; Head-Gordon, M. Use of the rVV10 nonlocal correlation functional in the B97M-V 
density functional: defining B97M-rV and related functionals. J. Phys. Chem. Lett. 2016, 8, 35-40.

(67) Wellendorff, J.; Lundgaard, K. T.; Jacobsen, K. W.; Bligaard, T. mBEEF: An accurate semi-local Bayesian error estimation density functional. J. Chem. Phys. 2014, 140, 144107.

(68) Zhao, Y.; Truhlar, D. G. A new local density functional for main-group thermochemistry, transition metal bonding, thermochemical kinetics, and noncovalent interactions. J. Chem. Phys. 2006, 125, 194101.

(69) Yu, H. S.; He, X.; Truhlar, D. G. MN15-L: A new local exchange-correlation functional for Kohn-Sham density functional theory with broad accuracy for atoms, molecules, and solids. J. Chem. Theory Comput. 2016, 12, 1280-1293.

(70) Becke, A. D. Densityfunctional thermochemistry. III. The role of exact exchange. J. Chem. Phys. 1993, 98, 5648-5652.

(71) Adamo, C.; Barone, V. Toward reliable density functional methods without adjustable parameters: The PBE0 model. J. Chem. Phys. 1999, 110, 6158-6170.

(72) Staroverov, V. N.; Scuseria, G. E.; Tao, J.; Perdew, J. P. Comparative assessment of a new nonempirical density functional: Molecules and hydrogen-bonded complexes. J. Chem. Phys. 2003, 119, 12129-12137.

(73) Zhao, Y.; Truhlar, D. G. The M06 suite of density functionals for main group thermochemistry, thermochemical kinetics, noncovalent interactions, excited states, and transition elements: two new functionals and systematic testing of four M06-class functionals and 12 other functionals. Theor. Chem. Acc. 2008, 120, 215-241.

(74) Wang, Y.; Verma, P.; Jin, X.; Truhlar, D. G.; He, X. Revised M06 density functional 
for main-group and transition-metal chemistry. Proc. Natl. Acad. Sci. U. S. A. 2018, 115, 10257-10262.

(75) Sun, J.; Perdew, J. P.; Ruzsinszky, A. Semilocal density functional obeying a strongly tightened bound for exchange. Proc. Natl. Acad. Sci. U. S. A. 2015, 112, 685-689.

(76) Hui, K.; Chai, J.-D. SCAN-based hybrid and double-hybrid density functionals from models without fitted parameters. J. Chem. Phys. 2016, 144, 044114.

(77) Chai, J.-D.; Head-Gordon, M. Long-range corrected hybrid density functionals with damped atom-atom dispersion corrections. Phys. Chem. Chem. Phys. 2008, 10, 66156620.

(78) Lin, Y.-S.; Li, G.-D.; Mao, S.-P.; Chai, J.-D. Long-range corrected hybrid density functionals with improved dispersion corrections. J. Chem. Theory Comput. 2012, 9, 263-272.

(79) Mardirossian, N.; Head-Gordon, M. wB97X-V: A 10-parameter, range-separated hybrid, generalized gradient approximation density functional with nonlocal correlation, designed by a survival-of-the-fittest strategy. Phys. Chem. Chem. Phys. 2014, 16, 9904-9924.

(80) Peverati, R.; Truhlar, D. G. Improving the accuracy of hybrid meta-GGA density functionals by range separation. J. Phys. Chem. Lett. 2011, 2, 2810-2817.

(81) Verma, P.; Wang, Y.; Ghosh, S.; He, X.; Truhlar, D. G. Revised M11 ExchangeCorrelation Functional for Electronic Excitation Energies and Ground-State Properties. J. Phys. Chem. A 2019, 123, 2966-2990.

(82) Krukau, A. V.; Vydrov, O. A.; Izmaylov, A. F.; Scuseria, G. E. Influence of the exchange screening parameter on the performance of screened hybrid functionals. $J$. Chem. Phys. 2006, 125, 224106. 
(83) Henderson, T. M.; Janesko, B. G.; Scuseria, G. E. Generalized gradient approximation model exchange holes for range-separated hybrids. J. Chem. Phys. 2008, 128, 194105.

(84) Peverati, R.; Truhlar, D. G. Screened-exchange density functionals with broad accuracy for chemistry and solid-state physics. Phys. Chem. Chem. Phys. 2012, 14, $16187-16191$.

(85) Grimme, S. Semiempirical hybrid density functional with perturbative second-order correlation. J. Chem. Phys. 2006, 124, 6158.

(86) Zhang, Y.; Xu, X.; Goddard, W. A. Doubly hybrid density functional for accurate descriptions of nonbond interactions, thermochemistry, and thermochemical kinetics. Proc. Natl. Acad. Sci. U. S. A. 2009, 106, 4963-4968.

(87) Zhang, I. Y.; Xu, X.; Jung, Y.; Goddard, W. A. A fast doubly hybrid density functional method close to chemical accuracy using a local opposite spin ansatz. Proc. Natl. Acad. Sci. U. S. A. 2011, 108, 19896-19900.

(88) Brmond, E.; Adamo, C. Seeking for parameter-free double-hybrid functionals: The PBE0-DH model. J. Chem. Phys. 2011, 135, 024106.

(89) Goerigk, L.; Grimme, S. Efficient and accurate double-hybrid-meta-GGA density functionals- evaluation with the extended GMTKN30 database for general main group thermochemistry, kinetics, and noncovalent interactions. J. Chem. Theory Comput. 2011, 7, 291-309.

(90) Kozuch, S.; Martin, J. M. L. Spin-component-scaled double hybrids: An extensive search for the best fifth-rung functionals blending DFT and perturbation theory. $J$. Comput. Chem. 2013, 34, 2327-2344.

(91) Mardirossian, N.; Head-Gordon, M. Survival of the most transferable at the top of 
Jacob's ladder: Defining and testing the $\omega \mathrm{B} 97 \mathrm{M}(2)$ double hybrid density functional. J. Chem. Phys. 2018, 148, 241736.

(92) Rappoport, D.; Furche, F. Property-optimized Gaussian basis sets for molecular response calculations. J. Chem. Phys. 2010, 133, 134105.

(93) Gill, P. M.; Johnson, B. G.; Pople, J. A. A standard grid for density functional calculations. Chem. Phys. Lett. 1993, 209, 506-512.

(94) Akima, H. A new method of interpolation and smooth curve fitting based on local procedures. J. Assoc. Comput. Mach. 1970, 17, 589-602.

(95) Shao, Y.; Gan, Z.; Epifanovsky, E.; Gilbert, A. T.; Wormit, M.; Kussmann, J.; Lange, A. W.; Behn, A.; Deng, J.; Feng, X.; Ghosh, D.; Goldey, M.; Horn, P. R.; Jacobson, L. D.; Kaliman, I.; Khaliullin, R. Z.; Kuś, T.; Landau, A.; Liu, J.; Proynov, E. I.; Rhee, Y. M.; Richard, R. M.; Rohrdanz, M. A.; Steele, R. P.; Sundstrom, E. J.; Woodcock, H. L.; Zimmerman, P. M.; Zuev, D.; Albrecht, B.; Alguire, E.; Austin, B.; Beran, G. J.; Bernard, Y. A.; Berquist, E.; Brandhorst, K.; Bravaya, K. B.; Brown, S. T.; Casanova, D.; Chang, C. M.; Chen, Y.; Chien, S. H.; Closser, K. D.; Crittenden, D. L.; Diedenhofen, M.; Distasio, R. A.; Do, H.; Dutoi, A. D.; Edgar, R. G.; Fatehi, S.; Fusti-Molnar, L.; Ghysels, A.; Golubeva-Zadorozhnaya, A.; Gomes, J.; HansonHeine, M. W.; Harbach, P. H.; Hauser, A. W.; Hohenstein, E. G.; Holden, Z. C.; Jagau, T. C.; Ji, H.; Kaduk, B.; Khistyaev, K.; Kim, J.; Kim, J.; King, R. A.; Klunzinger, P.; Kosenkov, D.; Kowalczyk, T.; Krauter, C. M.; Lao, K. U.; Laurent, A. D.; Lawler, K. V.; Levchenko, S. V.; Lin, C. Y.; Liu, F.; Livshits, E.; Lochan, R. C.; Luenser, A.; Manohar, P.; Manzer, S. F.; Mao, S. P.; Mardirossian, N.; Marenich, A. V.; Maurer, S. A.; Mayhall, N. J.; Neuscamman, E.; Oana, C. M.; Olivares-Amaya, R.; Oneill, D. P.; Parkhill, J. A.; Perrine, T. M.; Peverati, R.; Prociuk, A.; Rehn, D. R.; Rosta, E.; Russ, N. J.; Sharada, S. M.; Sharma, S.; Small, D. W.; Sodt, A.; Stein, T.; Stück, D.; Su, Y. C.; Thom, A. J.; Tsuchimochi, T.; Vanovschi, V.; Vogt, L.; Vy- 
drov, O.; Wang, T.; Watson, M. A.; Wenzel, J.; White, A.; Williams, C. F.; Yang, J.; Yeganeh, S.; Yost, S. R.; You, Z. Q.; Zhang, I. Y.; Zhang, X.; Zhao, Y.; Brooks, B. R.; Chan, G. K.; Chipman, D. M.; Cramer, C. J.; Goddard, W. A.; Gordon, M. S.; Hehre, W. J.; Klamt, A.; Schaefer, H. F.; Schmidt, M. W.; Sherrill, C. D.; Truhlar, D. G.; Warshel, A.; Xu, X.; Aspuru-Guzik, A.; Baer, R.; Bell, A. T.; Besley, N. A.; Chai, J. D.; Dreuw, A.; Dunietz, B. D.; Furlani, T. R.; Gwaltney, S. R.; Hsu, C. P.; Jung, Y.; Kong, J.; Lambrecht, D. S.; Liang, W.; Ochsenfeld, C.; Rassolov, V. A.; Slipchenko, L. V.; Subotnik, J. E.; Van Voorhis, T.; Herbert, J. M.; Krylov, A. I.; Gill, P. M.; Head-Gordon, M. Advances in molecular quantum chemistry contained in the Q-Chem 4 program package. Mol. Phys. 2015, 113, 184-215.

(96) Taylor, D. E.; ngyn, J. G.; Galli, G.; Zhang, C.; Gygi, F.; Hirao, K.; Song, J. W.; Rahul, K.; Anatole von Lilienfeld, O.; Podeszwa, R.; Bulik, I. W.; Henderson, T. M.; Scuseria, G. E.; Toulouse, J.; Peverati, R.; Truhlar, D. G.; Szalewicz, K. Blind test of density-functional-based methods on intermolecular interaction energies. J. Chem. Phys. 2016, 145, 124105.

(97) Neese, F.; Schwabe, T.; Grimme, S. Analytic derivatives for perturbatively corrected double hybrid density functionals: theory, implementation, and applications. J. Chem. Phys. 2007, 126, 124115.

(98) Fetisov, E. O.; Shah, M. S.; Long, J. R.; Tsapatsis, M.; Siepmann, J. I. First principles Monte Carlo simulations of unary and binary adsorption: CO2, N2, and $\mathrm{H} 2 \mathrm{O}$ in $\mathrm{Mg}$ MOF-74. Chem. Commun. 2018, 54, 10816-10819.

(99) Fang, H.; Kamakoti, P.; Ravikovitch, P. I.; Aronson, M.; Paur, C.; Sholl, D. S. First principles derived, transferable force fields for CO2 adsorption in Na-exchanged cationic zeolites. Phys. Chem. Chem. Phys. 2013, 15, 12882-12894.

(100) Fang, H.; Demir, H.; Kamakoti, P.; Sholl, D. S. Recent developments in first-principles 
force fields for molecules in nanoporous materials. J. Mater. Chem. A 2014, 2, 274291.

(101) Becker, T. M.; Heinen, J.; Dubbeldam, D.; Lin, L.-C.; Vlugt, T. J. Polarizable force fields for $\mathrm{CO} 2$ and $\mathrm{CH} 4$ adsorption in M-MOF-74. J. Phys. Chem. C 2017, 121, 4659-4673.

(102) Dubbeldam, D.; Walton, K. S.; Vlugt, T. J.; Calero, S. Design, parameterization, and implementation of atomic force fields for adsorption in nanoporous materials. Adv. Theory Simul. 2019, 2, 1900135. 


\section{Graphical TOC Entry}

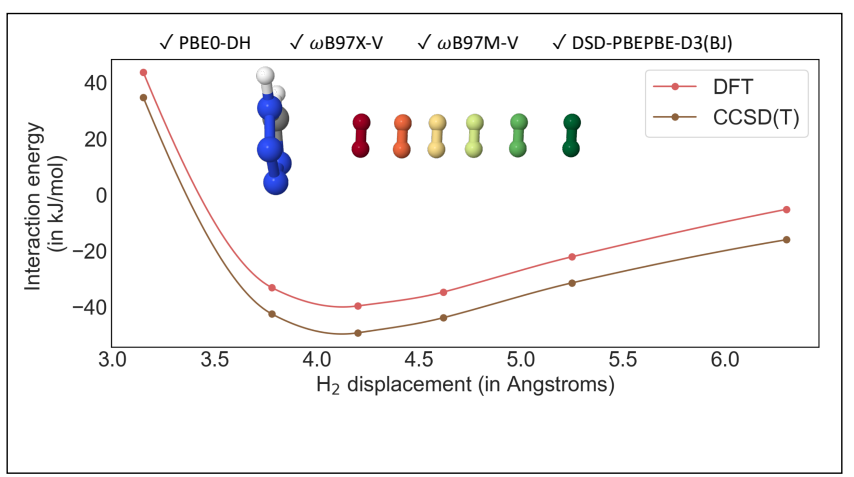

